\title{
Polarimetry of southern peculiar early-type $\operatorname{stars}^{\star}$
}

\author{
R.V. Yudin ${ }^{1}$ and A. Evans ${ }^{2}$ \\ 1 Central Astronomical Observatory of the Russian Academy of Sciences at Pulkovo, 196140 Saint-Petersburg, Russia \\ e-mail: ruslan@pulkovo.spb.su \\ 2 Department of Physics, Keele University, Keele, Staffordshire, ST5 5BG, UK
}

Received June 10, 1997; accepted February 2, 1998

\begin{abstract}
We present and discuss optical polarimetry of a large group (60 objects) of peculiar early-type stars (Herbig Ae/Be stars and candidate members, B [e] stars, extreme emission line objects etc.). Most were taken from "A new catalogue of members and candidate members of Herbig Ae/Be stellar group" The et al. (1994). For 40 out of the 60 objects polarization was measured for the first time. On the basis of the data we draw conclusions about the circumstellar shell configuration and orientation for several objects. Polarimetric variability on different timescales (minutes ... hours ... days) was investigated, as well as comparison with earlier measurements to investigate variability over longer time-scales. No circular polarization was detected to a $5 \sigma$ level in most of the investigated stars. Polarimetric data obtained here as well as data available from the literature were analyzed from the point of investigating the evolutionary status of the objects. The relationship between the polarimetric and photometric data was also investigated.
\end{abstract}

Key words: polarization - circumstellar matter: stars emission-line, Be - stars: pre-main sequence

\section{Introduction}

The first list of young early-type stars consisting of 26 objects was published 35 years ago by Herbig (1960). Later new lists of so-called Herbig Ae/Be (HAEBE) stars were suggested by Finkenzeller \& Mundt (1984)(55 objects), Shevchenko (1989) (87 objects) and recently by The et al. (1994) (287 HAEBE stars and related objects). During the last 35 years numerous spectroscopic and photometric investigations for individual HAEBE stars, as well as for

Send offprint requests to: R.V. Yudin

* Tables 6 to 22 are only available in electronic form at the CDS via anonymous ftp to cdsarc.u-strasbg.fr (130.79.128.5) or via http://cdsarc.u-strasbg.fr/Abstract.html the representative groups of these stars, have been carried out (see for example The et al. (1994). Unfortunately polarimetric investigations of young early-type stars are very limited. Up till 15 years ago only three attempts to study the polarimetric characteristics of HAEBE stars based on the original Herbig list - were made (Vrba 1975; Garrison \& Anderson 1978; Vrba et al. 1979).

In the following 10 years no polarimetric observations of groups of young early type stars were reported, except for the paper by Petrova \& Shevchenko (1987)(14 objects). In 1986 Grinin and collaborators started a program of simultaneous polarimetric and UBVRI photometry of photometrically active HAEBE stars with Algol-like minima (Grinin et al. 1988; Voshinnikov \& Grinin 1988; Berdyugin et al. 1990,1992 etc.). In the $1990 \mathrm{~s}$ polarimetric studies of HAEBE stars were reported by Jain et al. (1990), Hutchinson et al. (1994) and more intensively by Jain et al. (1995), but these observations also included only limited lists of HAEBE stars (8, 11 and 24 respectively). In relation to the total number of HAEBE stars and candidate members The et al. (1994), the number of HAEBE stars which have now be investigated polarimetrically is small $(\approx 30 \%$; Yudin 1988, 1992). Moreover, with few exceptions, no circular polarization measurements have been carried out for a sample of HAEBE stars to date. This deficit of polarimetric data does not allow us to judge the polarimetric characteristics of HAEBE stars as a definite group of young objects.

Polarimetric variability is detected in most investigated HAEBE stars (Vrba 1975; Garrison \& Anderson 1978; Vrba et al. 1979). Some of the objects show variability on a long time-scale: years, months (Scarrott et al. 1989; Jain et al. 1990, 1995). Scarrott et al. (1989) found changes in position angle (PA) for R Mon on a time-scale of years and assumed that they were caused by the precession of the circumstellar (CS) disc around the object. Other HAEBE stars with Algol-like minima show variability on a time-scale of days and an increase in the degree of polarization is accompanied by a decrease in visual flux 
Table 1. Herbig Ae/Be stars and candidate members included in the present study

\begin{tabular}{|c|c|c|c|c|c|c|c|}
\hline Name & Other name & $\alpha_{1950.0}$ & $\delta_{1950.0}$ & Spectral class & $V$ (mag) & $L$ (mag) & Table No. \\
\hline V1012 Ori & & 50905.5 & -022624 & B:e & $12.3-16.5$ & & 6 \\
\hline HD 34282 & $\mathrm{BD}-9^{\circ} 1110$ & 51338.09 & -095151.3 & $\mathrm{~A} 0 \mathrm{Ve}+\mathrm{sh}$ & $9.7-11.2$ & & 6 \\
\hline V346 Ori & HD 287841 & 52207.9 & +014057 & A5IIIe & $9.8-11.8$ & 6.8 & 6 \\
\hline HD 35929 & $\mathrm{BD}-08^{\circ} 1128$ & 52518.74 & $\begin{array}{lll}-08 & 22 & 04.4\end{array}$ & A5e & 8.2 & & 6 \\
\hline RY Ori & & 52939.57 & -025155.6 & F6/8ep & $11.1-13.1$ & 7.6 & 6 \\
\hline V586 Ori & $\mathrm{HD} 37258, \mathrm{BD}-06^{\circ} 1257$ & 53431.72 & $\begin{array}{lll}-06 & 11 & 02.2\end{array}$ & $\mathrm{~A} 2 \mathrm{Ve}$ & $9.1-10.8$ & 6.7 & 6 \\
\hline HD 37357 & $\mathrm{BD}-06^{\circ} 1264, \mathrm{KMS} 27$ & 53521.1 & -064413 & $\mathrm{~A} 0 \mathrm{Ve}$ & 8.86 & 6.33 & 7 \\
\hline HD 37411 & $\mathrm{BD}-05^{\circ} 1346$ & 53547.05 & -052653.4 & B9Ve & $9.8-9.9$ & 6.7 & 7 \\
\hline V599 Ori & KMS 49 & 53633.3 & $-07 \quad 1822$ & $\mathrm{~A} 5 / \mathrm{F} 0 \mathrm{e}$ & $13.5->16$ & 7.0 & 7 \\
\hline V350 Ori & & 53749.32 & -094341.9 & $\mathrm{~A} 0 \mathrm{e}$ & $10.8-13.2$ & 6.8 & 7 \\
\hline MWC 120 & $\mathrm{HD} 37806, \mathrm{BD}-02^{\circ} 1344$ & 53831.72 & -024428.8 & $\mathrm{~B} 9 \mathrm{Ve}+\mathrm{sh}$ & $7.9-8.0$ & 4.27 & 7 \\
\hline MWC 137 & & 61553.51 & +151809.1 & B0ep & $11.2-12.9$ & 5.1 & 14 \\
\hline VY Mon & & 62821 & +102814.9 & $\mathrm{~B} 8 \mathrm{e}$ & $12.7-14.9$ & 3.0 & 6 \\
\hline MWC 148 & $\mathrm{HD} 259440, \mathrm{BD}+05^{\circ} 1291$ & 63018.74 & +055019.2 & B0/6ep & $9.1-9.7$ & 6.0 & 9 \\
\hline GU CMa & HD 52721, MWC 164 & 65928.56 & $\begin{array}{lll}-11 & 13 & 41.4\end{array}$ & B2Vne & $6.5-7.0$ & $5.9-6.0$ & 7 \\
\hline $\mathrm{LkH} \alpha 218$ & & 70024 & -1121 & $\mathrm{~B} 6 \mathrm{e}$ & $11.9-12.2$ & $8.5(K)$ & 8 \\
\hline Z CMa & HD 53179, MWC 165 & 70122.52 & -112836 & B5/8neq+sh & $8.8-10.5$ & $1.9-2.2$ & 8 \\
\hline NX Pup & He $3-32, C D-44^{\circ} 3318$ & 71756.5 & -442935 & A0/F2IIIe & $9.3-11.0$ & $4.3-4.5$ & 8 \\
\hline RCW 34 & & 85440 & -425500 & O9 & 11.8 & 8.0 & 8 \\
\hline Не $3-331$ & HD 85567, WRA $15-452$ & 94859.38 & -604358.4 & B5Vne & $8.0-8.6$ & 7.2 & 13 \\
\hline He $3-515$ & HD 94509, WRA $15-677$ & 105125.02 & -580925.6 & $\mathrm{~A} 0 \mathrm{Ve}$ & 9 & & 11 \\
\hline He 3-554 & HD 95881 & 110015.0 & -711443 & $\mathrm{~A} 1 / 2 \mathrm{III} / \mathrm{IVe}$ & $7.8-8.4$ & & 10 \\
\hline Нe $3-597$ & HD 97048 & 110639.61 & $\begin{array}{lll}-77 & 23 & 01.2\end{array}$ & $\mathrm{~B} 9.5 / \mathrm{A} 0 \mathrm{ep}+\mathrm{sh}$ & $8.4-9.0$ & $4.55-4.95$ & 10 \\
\hline He 3-644 & HD 98922 & 112013.05 & -530549.3 & B9Ve & $6.7-6.8$ & 2.8 & 11 \\
\hline $\mathrm{He} 3-672$ & HD 100546 & 113114.13 & -695506.9 & B9Ve & $6.7-6.8$ & 4.0 & 11 \\
\hline Нe 3-692 & HD 101412 & 113721.61 & -595349.0 & $\mathrm{~B} 9.5 \mathrm{Ve}$ & 8.6 & & 11 \\
\hline He $3-741$ & HD 104237 & 115733.47 & -775450.5 & $\mathrm{~A} 4 \mathrm{e}$ & $6.5-6.6$ & 3.05 & 17 \\
\hline HD 132947 & $\mathrm{CPD}-62^{\circ} 4379$ & $1500 \quad 48.37$ & -625611.6 & $\mathrm{~A} 0 \mathrm{e}$ & 8.9 & & 12 \\
\hline HD 141569 & $\mathrm{BD}-03^{\circ} 3833$ & $15 \quad 4720.21$ & -034611.9 & e & $6.9-7.1$ & $6.8(J)$ & 12 \\
\hline HD 142666 & $\mathrm{BD}-21^{\circ} 4228$ & 155343.26 & -215300.0 & $\mathrm{~A} 7 / 8 \mathrm{Ve}$ & $8.6-9.8$ & & 10 \\
\hline RY Lup & & 155605 & -4013.6 & GOVe & $10.2-13.1$ & $5.9-6.2$ & 14 \\
\hline MWC 863 & HD 150193 & $1637 \quad 16.45$ & -234756.4 & $\mathrm{~A} 0 / 4 \mathrm{Ve}$ & $8.6-8.9$ & $4.2-4.3$ & 13 \\
\hline KK Oph & He $3-1346$, AS 220 & 170700.73 & -271136.2 & $\mathrm{~A} 5 \mathrm{Ve}$ & $10.3-12.85$ & 4.0 & 17 \\
\hline HD 163296 & He $3-1524$, MWC 275 & 175320.65 & -215657.0 & $\mathrm{~A} 0 / 2 \mathrm{Vep}+\mathrm{sh}$ & $6.8-6.9$ & $3.5-3.6$ & $15-17$ \\
\hline $\mathrm{LkH} \alpha 112$ & & 180116.0 & -242236 & B3e & $9.6-10.2$ & & 9 \\
\hline $\mathrm{LkH} \alpha 115$ & & 180138.9 & -242620 & B9e & $11.9-12.2$ & & 12 \\
\hline VV Ser & & 182614.33 & +000639.6 & $\mathrm{~B} 1-\mathrm{A} 2 \mathrm{e}$ & $11.6-12.8$ & $4.5-4.8$ & 11 \\
\hline $\mathrm{S} \mathrm{CrA}$ & & 185747.6 & -370122 & $\mathrm{G}: \mathrm{V}: \mathrm{e}$ & $10.6-13.2$ & $4.7-5.1$ & 13 \\
\hline V1295 Aql & HD 190073, MWC 325 & 200034.43 & +053549.6 & AoIVep+sh & 7.9 & 4.8 & 8 \\
\hline
\end{tabular}

(Grinin 1994). Such variations are explained in terms of nonperiodic eclipse of stellar light by rotating "protoplanetary" condensations. Note that, in all the above cases, the main origin of polarization is scattering by dust grains in CS shells. However, it is well known that HAEBE stars are also surrounded by extended and dense gas shells, which may also contribute to the observed polarization. The short time-scale polarimetric variability which has been established for a few HAEBE stars (Dzhakusheva et al. 1988; Beskrovnaya et al. 1995) can be explained by Thomson scattering. Unfortunately up to now we have had no information on polarimetric variability on time-scales of hours or less, except for the few cases mentioned above. Thus, the investigation of polarimetric variability on different time-scales might give us additional information on the mechanisms of polarization.

Bastien $(1985,1988)$ showed that there exists a correlation between the polarization and infrared (IR) colour indices for young T Tau stars (TTS). More detailed investigations of such correlations were carried out by Yudin (1988) for a sample of TTS and HAEBE stars. He showed that a strong linear correlation exists between $\log p$ and IR excess for all types of young stars of different spectral classes. One of the conclusions from Yudin's (1988) work was that the above mentioned correlations can be used for selecting stars at the pre-main sequence (PMS) stage of evolution. However, deviation of an object's position from the above correlations for young stars can be caused not only by the different evolutionary status of objects but also by different orientations of disc-like dust structures around the stars.

A further important factor is the relation of HAEBE stars with other groups of peculiar early type stars, such as $\mathrm{Be}$ and $\mathrm{B}[\mathrm{e}]$ stars, luminous blue variables (LBVs) etc. The evolutionary status of a significant group of $\mathrm{B}[\mathrm{e}]$ and Be stars is controversial; are they PMS stars, or at a later stage of evolution (see e.g. the investigation of the $\mathrm{B}[\mathrm{e}]$ star MWC 349 (Yudin 1995))? In many cases polarimetric properties of HAEBE stars and $\mathrm{B}[\mathrm{e}](\mathrm{Be})$ stars are similar.

Taking into account all of the above factors we have carried out a polarimetric study of peculiar early-type stars with the following aims:

1. First, to obtain new polarimetric (linear and circular) data for a large group of HAEBE stars and candidate members, as well as for some peculiar Be and $\mathrm{B}[\mathrm{e}]$ stars and LBVs. The stars observed in the present programme are listed in Tables 1, 2. Table 1 contains HAEBE stars and candidate members, while Table 2 
Table 2. B $[\mathrm{e}]$ stars, extreme emission line objects and other early-type emission line stars with IR excess included in the present study

\begin{tabular}{|c|c|c|c|c|c|c|c|}
\hline Name & Other name & $\alpha_{1950.0}$ & $\delta_{1950.0}$ & Spectral class & $V(\mathrm{mag})$ & $L$ (mag) & Table No. \\
\hline HD 290770 & $\mathrm{BD}-01^{\circ} 978$ & 53430.61 & -013907.1 & $\mathrm{~B} 8 \mathrm{e}$ & $9.2-9.3$ & & 19 \\
\hline AS 117 & $\mathrm{BD}-14^{\circ} 1319$ & 60121.9 & -145252 & $\mathrm{Ae}$ & $9.4-10$ & & 18 \\
\hline AS 119 & & $\begin{array}{lll}6 & 07 & 02.27\end{array}$ & -093814 & $?$ & 11 & & 19 \\
\hline MWC 158 & HD $50138, \mathrm{BD}-06^{\circ} 1775$ & 64907.58 & -065421.6 & $\mathrm{~B} 6 \mathrm{~V}[\mathrm{e}]+\mathrm{sh}$ & $6.6-6.8$ & $2.7-2.95$ & 17 \\
\hline EW CMa & HD 56014, MWC 170 & 71212.83 & $-2615 \quad 54.1$ & B3IIIep & $4.3-4.7$ & 4.08 & 18 \\
\hline MWC 843 & HD $59319, \mathrm{BD}-21^{\circ} 1955$ & 72627.99 & -215136.4 & $\mathrm{~B} 8 \mathrm{Ve}$ & $8.7-8.8$ & & 19 \\
\hline AS 160 & Не $3-52$ & 73700.3 & -243806 & $\mathrm{~B}[\mathrm{e}]$ & $11.2-11.4$ & 5.9 & 19 \\
\hline 3 Pup & L Pup, HD 62623 & 74147.96 & $-28 \quad 50 \quad 03.2$ & A2/3Iabe & $3.9-4.5$ & 0.8 & 18 \\
\hline Hilt 641 & HD 64315 & 75015.87 & -261758.9 & $\mathrm{O} 6 \mathrm{e} / \mathrm{A} 1 \mathrm{Iae}$ & $9.16-9.24$ & & 20 \\
\hline He $3-140$ & $\mathrm{CD}-49^{\circ} 3441$ & 81253.4 & -500034 & $\mathrm{~B} 8 \mathrm{e}$ & 9.3 & & 19 \\
\hline He $3-180$ & WRA $15-238$ & 83333.0 & -402605 & $\mathrm{~B} 8 \mathrm{e}$ & 10.7 & & 19 \\
\hline He $3-365$ & HD 87643, MWC 198 & 100249.72 & $\begin{array}{lll}-58 & 25 & 16.2\end{array}$ & $\mathrm{~B} 3 / 4[\mathrm{e}]$ & $8.5-9.1$ & $1.98-2.08$ & 21 \\
\hline WRA $15-535$ & He $3-394$ & 101335.3 & -573642 & $\mathrm{~B} 5 / 6 \mathrm{e}$ & 10.5 & 4.7 & 22 \\
\hline HD 90578 & He $3-418$ & 102409.05 & -573419.3 & $\mathrm{~B} 1 \mathrm{Ie} / \mathrm{IIe}$ & $9.26-9.36$ & & 21 \\
\hline HD 303004 & $\mathrm{CD}-58^{\circ} 3348$ & 103146.1 & -584546 & $\mathrm{Be}$ & $10.0-11.8$ & & 22 \\
\hline WRA $15-689$ & $\mathrm{CPD}-59^{\circ} 2854$ & 105354 & -595819 & $\mathrm{~B} 2 / 3 \mathrm{Ve}$ & $10.0-10.5$ & & 19 \\
\hline MCW 1264 & HD 152291 & 165055.64 & -403419.2 & B2II:e & $8.5-8.8$ & & 12 \\
\hline MWC 257 & HD $156702, \mathrm{He} 3-1384$ & 171724.27 & -383611.3 & $\mathrm{~B} 3 / 5 \mathrm{e}$ & $8.7-8.8$ & & 18 \\
\hline MWC 272 & HD 316285, He $3-1482$ & $1745 \quad 04.82$ & -275955.2 & $\mathrm{~B} 2 / 3[\mathrm{e}]$ & $9.0-9.2$ & 2.96 & 21 \\
\hline MWC 900 & HD 164246, He $3-1554$ & 175835.78 & -393930.0 & $\mathrm{~B} 8 / 9 \mathrm{IVe}$ & $9.0-9.1$ & & 18 \\
\hline MWC 925 & HD 169142, He $3-1688$ & 182117.95 & -294828.1 & B9Ve & $8.0-8.13$ & 5.66 & 22 \\
\hline
\end{tabular}

contains B $[\mathrm{e}]$ stars, extreme emission-line objects and other early-type stars with IR excesses. In addition two TTS (S CrA and RY Lup) were included in our study. The whole list of programme stars consists of 60 objects located mainly in the southern hemisphere; most were taken from The et al. (1994). For 40 of the 60 objects polarimetric data have been obtained for the first time.

2. Second, to study polarimetric variability of the programme stars on different time-scales (minutes ... hours ... days). For this reason most of the investigated stars were observed at least twice. Some objects were observed repeatedly during a night and/or monitored during a period of a few hours. Also, to study variability on long time-scales, the data presented here were compared with previously published data.

3. Third, to compare the polarimetric characteristics for a significant group of HAEBE stars and related objects with their photometric data. It is well known that most HAEBE stars show significant IR excess due to CS dust (Berrilli et al. 1989; Hillenbrandt et al. 1992). However the configuration of CS shells around HAEBE stars is currently a point of discussion. At present it is not possible to conclude whether these shells have spherical symmetry or, perhaps, have a disc-like structure. In the case of optically thin discs any IR excess does not depend on the viewing aspect. On the other hand, if the main origin of polarization is scattering in a dust disc-like structure, the degree of polarization will strongly depend on the inclination of the discs (see e.g. McLean \& Brown 1978). For this reason we selected for our programme stars with different IR excesses in order to search for a link between polarization and IR excess. The range of observed flux in the $V$ and $L$ bands from the literature is given in Tables 1,2 .
In Sect. 3 data obtained for individual objects are briefly discussed. In some cases we present an estimate of brightness for individual objects based on our data. A statistical study of the polarimetric properties of the selected group of stars is presented in Sect. 4 . In Sect. 5 we discuss the relationship between the polarimetric and photometric characteristics of some objects and draw conclusions about the orientation of CS discs around them.

\section{Observations and analysis}

\subsection{Observations}

A programme of optical polarimetry of selected stars was carried out during three runs in 1994 August 2-15 (JD 2449571-2449580), 1995 January 23 - February 6 (JD 2449742-2449755) and 1995 July 18 - August 31 (JD2449917-2449930) at the South African Astronomical Observatory (SAAO). The University of Cape Town photometer-polarimeter module (Cropper 1985) was used on the SAAO $0.75 \mathrm{~m}$ telescope, although the observations for the period 1994 August 9-15 were carried out on the SAAO $1 \mathrm{~m}$ telescope. The polarimeter module contains a half-wave and quarter-wave plate rotating in opposite directions, allowing simultaneous linear and circular polarization measurements. During the first run of observations data in the Johnson $U B V$ and Cousins $R_{\mathrm{c}} I_{\mathrm{c}}$ filters were obtained. In the second and third runs only $U B V$ or $V R_{\mathrm{c}} I_{\mathrm{c}}$ filters were used respectively. All observations were made with a $20^{\prime \prime}$ aperture. The polarimeter was calibrated using the polarimetric standards of Hsu \& Breger (1982). The instrumental polarization is very small and has been neglected. It is $\lesssim 0.03 \%$ (linear) and $0.017 \pm 0.005 \%$ (circular), both in the $V$ band (Cropper 1985; D. Buckley, private communication); values in other wavebands are 
similar. The design of the instrument (see Cropper 1985) ensures that there is no significant leakage of linear to circular polarization.

Data for individual objects are presented in Tables 622. The columns in these tables give the Julian Date of the middle of each observation, the filter used, the basic integration time for the corresponding filter, the number of integrations from which the values were obtained, the linear polarization and its standard error (both in percent), the equatorial PA and its standard error (both in degrees), the internal errors $\left(\sigma_{1}\right)$ in $\Theta$, which were calculated from photon counting statistics, the normalized Stokes parameters (NSPs) $U$ and $Q$ (both in percent), and the circular polarization and its standard error (both in percent).

\subsection{Errors in polarization}

Despite the fact that, as is well known, noise introduces a bias to determinations of the degree of polarization (Serkowski 1958, 1962), this fact seems largely to be ignored when new polarimetric results on young stars are presented (however see Whittet et al. 1992 and Martin et al. 1992 for exceptions), although it is often not mentioned explicitly even when it is taken into account (P. Bastien, private communication). In the papers by Whittet et al. (1992) and Martin et al. (1992), the data have been corrected for noise biasing using the analytical formula (Clarke \& Stewart 1986)

$p=p_{\text {obs }}\left[1-\left(\sigma / p_{\text {obs }}\right)^{2}\right]^{1 / 2}$,

where $\sigma$ is the standard deviation of the observed polarization $p_{\text {obs }}$. We present in Tables 6-22 biased data but in the analysis for the presence of polarization and polarimetric variability we used an estimate of the true polarization. Note however, that it does not affect the analysis of polarimetric variability, which was the prime aim of our study. For an estimate of errors in PA for the cases $p_{\text {obs }} / \sigma_{\mathrm{p}}>6$ we have used the simple Serkowski formula $\sigma_{\Theta}=28.65 \sigma_{\mathrm{p}} / p$. Naghizadeh-Khouei \& Clarke (1993) have shown that "below this critical value, the nonnormality of the statistics provides a situation for which error estimation is complex and cannot be considered in terms of the above formula". Therefore in the cases were $p_{\text {obs }} / \sigma_{\mathrm{p}}<6$ we present in the Tables $6-22$ a confidence interval for the PA at the $68.28 \%$ ( $1 \sigma)$ level (see NaghizadehKhouei \& Clarke 1993 for details). Besides, we also present in Tables 6-22 the internal errors $\sigma_{1}$, calculated from photon counting statistics. In addition note that we tabulate in the Tables 6-22 not only the values of $p$ and $\Theta$ but also the values of NSPs. The reason for this is that the values of $p$ and $\Theta$ are more useful for comparison with data published in previous papers, whereas for astrophysical studies of their statistical behaviour the analysis of NSPs is preferable (see Clarke \& Stewart 1986 for details). While the statistical tests for the presence of polarization and for changes of polarimetric parameters are valid only for normally distributed Stokes parameters, all the data were tested for normality by using the $\chi^{2}$-test. In any case we are confident that noise from scintillation is reduced to a very low level as a result of the rapid rotation of the waveplates.

\subsection{Value of polarization}

To investigate the presence of polarization for stars with low polarization, or low signal-to-noise in the polarization measurements, we used the method described by Clarke et al. (1985) or Clarke \& Stewart (1986); in the following we refer to this method as the "Clarke-Stewart test". The method adopted is connected with a projection on the $U-Q$ plane yielding a conservative estimate for the true polarization and allowing determination of confidence intervals. The confidence region for the true polarization is given by a circle of radius $\sigma \sqrt{z_{(1-\alpha)}}$ centered on $u, q$, where $z_{(1-\alpha)}=2(N-1)\left(\alpha^{-1 /(N-1)}-1\right)$ for a $(1-\alpha)$ confidence level, $N$ is the number of integrations, $u, q$ are estimates of the Stokes parameters and $\sigma$ is its standard error (see Clarke et al. 1985 for details). According to Clarke et al. (1985) "the source is said to be unpolarized if the origin is contained within the circle radius at the chosen $(1-\alpha)$ confidence level".

\subsection{Variability of polarization}

In order to investigate the variability of the polarization parameters we apply the statistical Welsh test, as recommended by Clarke \& Stewart (1986). When the data are intercompared, the Welsh test indicates that the mean values for Stokes parameters are significantly different at the $99 \%$ confidence level. Clarke \& Stewart (1986) have also pointed out that "Welsh procedures may result in a polarimetric difference being detected between sets of points when none of the individual points is polarized, because the Welsh test weights observations with respect to mean values and standard mean errors".

In the following section we briefly present and discuss the data obtained for individual stars, and compare our measurements with earlier results.

\section{Discussion of individual objects}

\subsection{Herbig Ae/Be stars and candidate members}

\subsubsection{V1012 Ori}

The range of the photographic variability of V1012 Ori is $12.5<m_{\mathrm{ph}}<16^{\mathrm{m}} \cdot 5$ : the object is a strong photometric variable. No polarimetric measurements have been published previously. In spite of the fact that our data indicate a degree of polarization for this object $p \approx 1 \%$ in 
the $U B V$ bands no polarization was in fact detected at the $95 \%$ confidence level. Note that our observations were carried out when the object was at $V \approx 12^{\mathrm{m}}$, so that it was observed close to maximum light. Additional observations close to minimum would be most interesting.

\subsubsection{HD 34282}

According to The et al. (1994) the variability range of HD 34282 in the visual is $9^{\mathrm{m}} \cdot 7<V<11^{\mathrm{m}} \cdot 2$ but Bogaert $\&$ Waelkens (1990) have pointed out that the amplitude of photometric variability of this star is about $3^{\mathrm{m}}$ in the $V$ band. Our polarization measurements were made when the object was brighter than $V \approx 10^{\mathrm{m}}$. The object has a low level of polarization, not exceeding $0.2 \%$ in the $V$ band. No statistically significant polarization was detected to $95 \%$ confidence in any of the measurements.

\subsubsection{V346 Ori (HD 287841)}

According to Manfroid et al. (1991, 1994) and Sterken et al. $(1993,1995)$ this object varies in $V$ between 9 m. 8 and $11^{\mathrm{m}} .8$. Photometric data from these papers indicate that it shows significant variability on a time-scale of days. The brightness of the object during the observations described here was about $10^{\mathrm{m}}$ (i.e. close to maximum light) and the degree of polarization does not exceed $0.6 \%$, with no evidence of variability at the $3 \sigma$ level. The Clarke-Stewart test shows the presence of polarization in the $V$ band at the $95 \%$ confidence level in only one measurement, on JD. . 9746. In all other measurements polarization was not detected. This is clear evidence of polarimetric variability in this star, and the presence of intrinsic polarization.

\subsubsection{HD 35929}

The degree of polarization of HD 35929 does not exceed $0.1 \%$. There is no statistically significant polarization at the $95 \%$ confidence level. Also, no polarimetric variability was detected for this object in four $V$-band measurements made on two nights. Miroshnichenko et al. (1997) supposed that HD 35929 is very close to the main sequence.

\subsubsection{RY Ori}

This object was considered by The et al. (1994) to be a F-type PMS star. The range of photographic variability of RY Ori is $10 \mathrm{~m} .8<m_{\mathrm{ph}}<13^{\mathrm{m}} .9$ and the star varies in $V$ between $11^{\mathrm{m}} .15$ and $13^{\mathrm{m}} .05$ (Manfroid et al. 1991, 1994 and Sterken et al. 1993, 1995. In our two measurements the star shows a large degree of polarization $(p \approx 4 \%-6 \%)$ when the star was at $V \approx 13.7$, near minimum. This degree of polarization is consistent with the large opticalIR colour index $3^{\mathrm{m}} \cdot 6<(V-L)_{\mathrm{obs}}<5^{\mathrm{m}} \cdot 4$. In spite of the large errors the Clarke-Stewart test shows the presence of polarization at the $95 \%$ level in the first measurement, but no polarization was detected in the second measurement taken 10 minutes later. Further polarimetric observations of the object with high accuracy are highly desirable.

\subsubsection{V586 Ori (HD 37258)}

The first polarimetric measurements of this star (Breger 1976; Vrba et al. 1987) showed a small degree of polarization $\left(p=0.27 \% \pm 0.10 \% \Theta=94^{\circ}\right.$ and $p=1.02 \% \pm 0.05 \%$ $\Theta=82^{\circ}$ respectively). Later this object was investigated by Berdyugin et al. (1993). They monitored this object for 40 nights and detected variation in flux from $V=9^{\mathrm{m}} .11$ to $V=10^{\mathrm{m}} .51$, with $p_{V}^{\max } \approx 3.4 \%$. The analysis of polarimetric and photometric variability allowed them to conclude that V586 Ori is a member of the HAEBE class with Algol-like minima. According to The et al. (1994) the lower level of its excursions in $V$ is $10^{\mathrm{m}} .75$. Furthermore the star has a large IR excess $2^{\mathrm{m}} \cdot 9<(V-L)_{\text {obs }}<4^{\mathrm{m}}$. 1 . The Algol-like fading epoch was predicted by Shevchenko et al. (1994) to occur in 1995. Our measurements, which were made in 1995 on three occasions show no evidence of polarimetric variability within the errors, both in degree of polarization and in PA. Besides the Clarke-Stewart test indicates no polarization at the $95 \%$ confidence level in any of our measurements. Possibly the star was observed near maximum light $\left(V \approx 9^{\mathrm{m}} .5\right)$ and low level of polarization.

\subsubsection{HD 37357}

This object has negligible degree of polarization with no evidence of variability. The Clarke-Stewart test shows no polarization at the $95 \%$ confidence level in our two measurements. The average visual magnitude $\left(8^{\mathrm{m}} .85\right)$ was in good agreement with earlier values (see Table 1).

\subsubsection{HD 37411}

According to Herbig (1994) this star lies close to the main sequence. Photometric variability has not previously been detected, and no polarimetric data have been published for this object. The Welsh test shows that the object is a polarimetric variable in all of the wavebands used. However the degree of polarization is small. The average values of polarization are as follows:

$$
\begin{array}{ll}
p_{U} \approx 0.8 \% & \Theta_{U} \approx 6^{\circ} \\
p_{B} \approx 0.4 \% & \Theta_{B} \approx 20^{\circ} \\
p_{V} \approx 0.5 \% & \Theta_{V} \approx 32^{\circ} .
\end{array}
$$

There may be a variation in PA from the $U$ to $V$ bands which, together with the variability of polarization, is evidence for intrinsic polarization. No photometric variability 


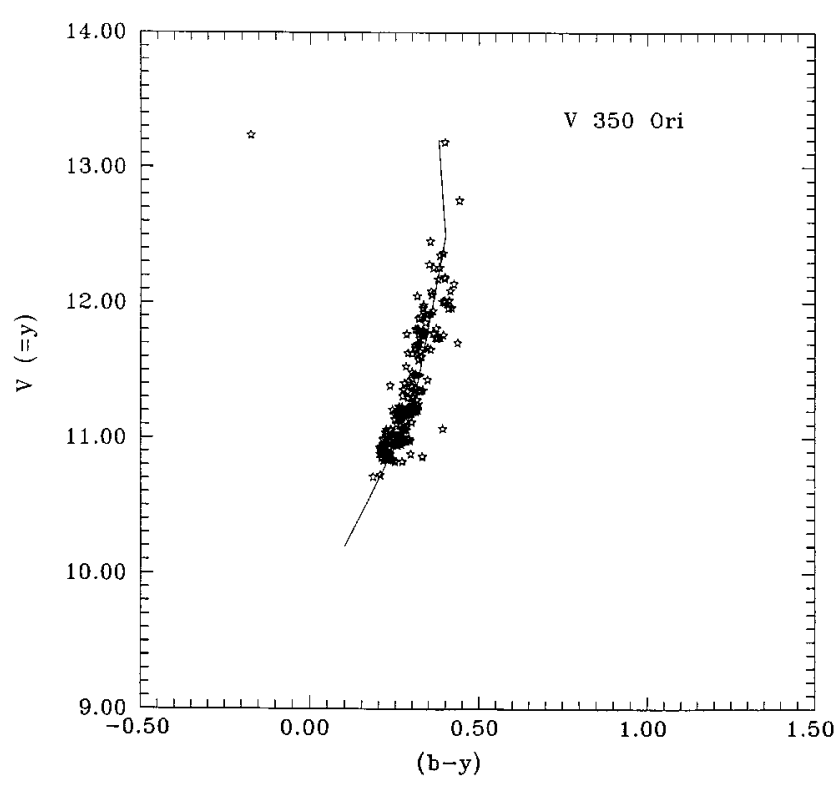

Fig. 1. Photometric behaviour of V350 Ori

was detected in our measurements and the photometric data $\left(V \approx 9^{\mathrm{m}} .8\right)$ are in good agreement with earlier results.

\subsubsection{V599 Ori}

The range of photographic variability of V599 Ori is $15^{\mathrm{m}} .1<m_{\mathrm{ph}}<16^{\mathrm{m}} .3$ (Kholopov 1985) and The et al. (1994) have measured $V \simeq 13^{\mathrm{m}} \cdot 47$; this object has a very large optical-IR colour index $(V-L)_{\text {obs }} \approx 6^{\mathrm{m}} \cdot 5$. No polarimetric data have been published previously. Our only $U B V$ measurement shows a large degree of polarization (about $5 \%$ in the $U$ and $B$ bands).

Note however that, in view of the large errors, the polarization was statistically detected at the $95 \%$ confidence level in the $U$ band only.

\subsubsection{V350 Ori}

According to The et al. (1994) the variability range of V350 Ori in the visual is $10.75<V<12^{\mathrm{m}}$.45 but measurements obtained in the long-term monitoring at ESO (Manfroid et al. 1991, 1994 and Sterken et al. 1993, 1995) went fainter than this $\left(V=13^{\mathrm{m}} \cdot 24\right)$. Taking into account data obtained at ESO we conclude that the photometric behaviour of the object is similar to the behaviour detected for HAEBE stars with Algol-like minima (Grinin 1992), namely the star was bluer at lower levels of flux. The photometric behaviour of V350 Ori and one HAEBE star with Algol-like minima (UX Ori) are shown in Figs. 1 and 2 .

During the observations described here strong photometric and polarimetric variability were detected in the

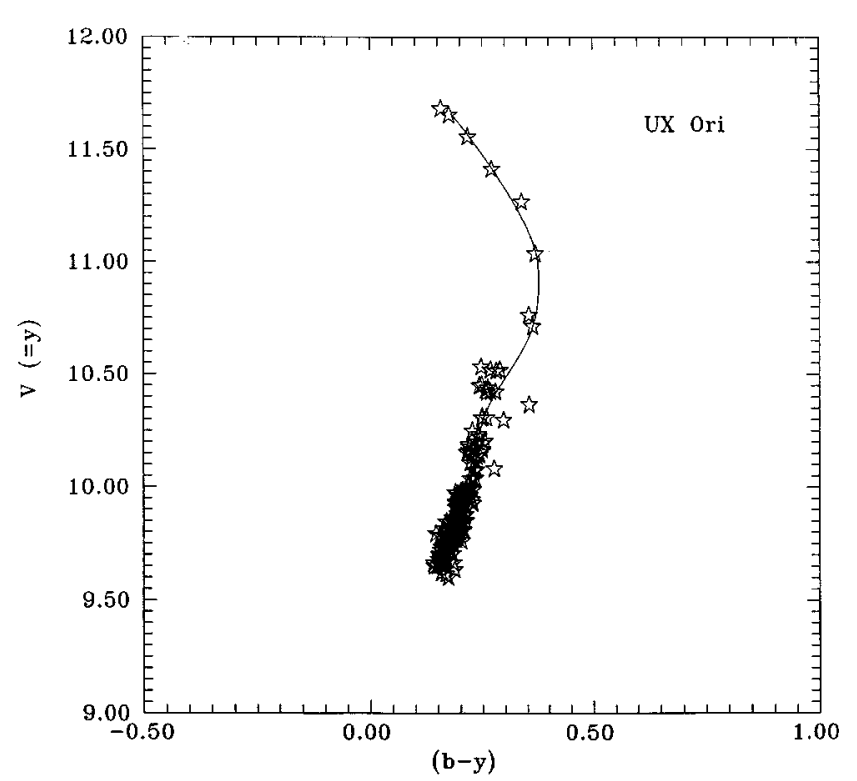

Fig. 2. Photometric behaviour of UX Ori

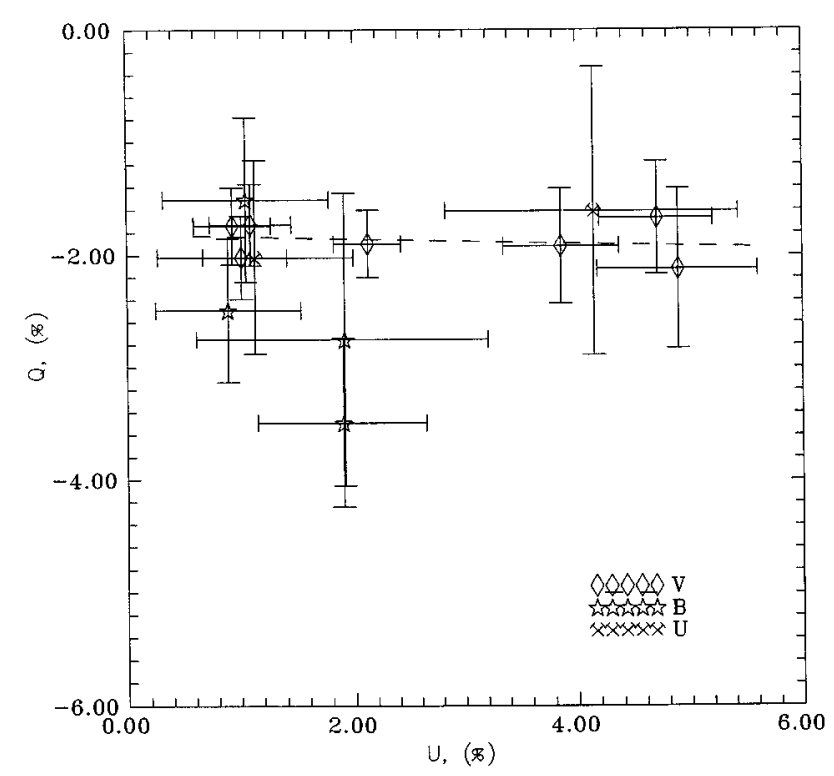

Fig. 3. $U-Q$ diagram for V350 Ori

sense that the polarization was high when the star was fainter. The variation of polarimetric parameters in the $U-Q$ plane are shown in Fig. 3.

On JD. . 9744 and JD. . 9747 the star was fainter by about $0.5-0^{\mathrm{m}} .7$ in all photometric bands than it was on JD. . 9749 and JD...9751. Note that the PA varied by about $20^{\circ}$ between the low and high levels of polarization. On the $U-Q$ diagram the data are concentrated along a line that can be interpreted as scattering of stellar radiation by dust blobs rotating around the star. From the above we conclude that V350 Ori is a new candidate member of the subgroup of HAEBE stars with Algol-like minima. 


\subsubsection{MWC 120 (HD 37806)}

According to The et al. (1994) this object has a small range of photometric variability $\left(7^{\mathrm{m}} \cdot 89<V<7^{\mathrm{m}} \cdot 98\right)$ and an optical-IR colour index $(V-L)_{\text {obs }} \approx 3^{\mathrm{m}} \cdot 6$. It is polarized at the $0.5 \%-0.7 \%$ level, with good accuracy. No polarimetric variability at the $3 \sigma$ level was detected in our measurements in the $U$ and $B$ bands, while the polarization in the $V$ band is variable on a time-scale of days at the $95 \%$ confidence level. There may be variation in the PA from the $U$ to the $V$ bands. Its polarization has a maximum in the $B$ band, and decreases in the $U$ and $V$ bands. The wavelength dependence of the PA suggests the presence of an intrinsic component of polarization. However, no photometric variability was detected, and the visual magnitude $\left(V \approx 7^{\mathrm{m}} \cdot 9\right)$ is in good agreement with the data of The et al. (1994).

\subsubsection{MWC 137}

According to Shevchenko (1989) this object is a very massive O-type star at a very early stage of evolution. Optical and mid-IR photometry for this object was published by Shevchenko (1989), Bergner et al. (1993) and Bergner et al. (1995). Heckert \& Zeilik (1981) found a significant degree of polarization in the IR (the degree of polarization in the JHK bands is about $2 \%-3 \%$ ). Optical polarimetry (in the $R$ band) was previously carried out by Yudin $(1994,1995)$, who found a high degree of polarization at the $6 \%-9 \%$ level, with large variability in both the PA and the degree of polarization. Furthermore Yudin (1995) noted that an increase in the degree of polarization is accompanied by an increase in flux in the $B$ band. Our data, obtained over a short time interval (three days), indicate a high degree of polarization (the average value of polarization in the $V$ band is $p_{V} \approx 5.50 \%$ and $\Theta_{V} \approx 160^{\circ}$ ) with no evidence of variability at the $95 \%$ confidence level. Further, no photometric variability was detected during our measurements in any of the filters used. Note that the data of Jain et al. (1995) (in their highest accuracy observation) indicate a significantly larger degree of polarization of MWC 137 in the $V$ band (about $6.56 \%$ ) with the same PA (about $161^{\circ}$ ). However the observational errors in both sets of observations were large.

\subsubsection{VY Mon}

An analysis of the photometric and spectroscopic characteristics of VY Mon led to the conclusion that the object is a member of FU Ori group of young stars (Miroshnichenko et al. 1992). The range of photographic variability is $13^{\mathrm{m}} \cdot 7-17^{\mathrm{m}} \cdot 4$ (Kholopov 1985). This star has one of the largest IR excesses $\left[(V-L)_{\text {obs }} \approx 10^{\mathrm{m}}\right]$. According to Pavlova \& Rspaev (1985) the polarization of VY Mon is about $10 \%$, based on two polarimetric measurements in the $B$ band. Later optical polarimetry (in the $R$ band) was carried out by Miroshnichenko et al. (1992). They also detected a large degree of polarization at the level of about $10 \%$, and found variations of about $2 \%$ and $10^{\circ}$ in PA on a time-scale $\sim$ days. Our data, together with data from Miroshnichenko et al. (1992), indicate that the polarization depends on the light excursions in the sense that the polarization is larger when the star is fainter. On JD. . .9751 the star was fainter by about 0.5 in comparison with our earlier measurements and the polarization in the $V$ band was much higher. This object has the largest polarization in our survey. Within the errors the present measurements generally agree with earlier results and also indicate variability on a time-scale of days, both in degree of polarization and in PA. A slight difference in our polarization data and those of Miroshnichenko et al. (1992) can be connected with the different visual fluxes when the measurements were carried out.

\subsubsection{MWC 148 (HD 259440)}

An analysis of previously published photometric and polarimetric measurements (Coyne et al. 1974; Serkowski et al. 1969; Hiltner 1956; Kilkenny et al. 1985) allows us to conclude that the brightness of this object in the $V$ band varies from $9^{\mathrm{m}} \cdot 1$ to $9^{\mathrm{m}} \cdot 7$ and the degree of polarization ranges between $3.3 \%$ and $4.05 \%$, with the PA in the range $163^{\circ}-166^{\circ}$. Our data also indicate a degree of polarization at the level of about $3 \%-4 \%$ and $\mathrm{PA} \approx 165^{\circ}$ but also show variability of the Stokes parameters at the $95 \%$ confidence level in the $V$ band on a time-scale of minutes. There is a possibility that the data trace an ellipse in the $U-Q$ diagram but the errors are large. We plan to discuss the data for this object in a separate paper.

\subsubsection{GU CMa (HD 52721)}

The polarization for this object is small $(\approx 1 \%)$. The average value of polarization in the $V$ band is about $p_{V} \approx 1.2 \%$ and $\Theta_{V} \approx 13^{\circ}$. Previous polarimetric data were obtained by Hall $(1958)\left(p \approx 1.4 \%, \Theta \approx 16^{\circ}\right)$, Voshchinnikov \& Marchenko $(1982)\left(p \approx 1.2 \%, \Theta \approx 19^{\circ}\right)$ and JB95 $\left(p_{V} \approx 1.3 \%, \Theta_{V} \approx 19^{\circ}\right)$. In addition three $U B V$ measurements of polarization were published by Serkowski et al. (1975), from which the mean values are:

$$
\begin{aligned}
& p_{U} \approx 1.1 \% \quad \Theta_{U} \approx 18^{\circ} \\
& p_{B} \approx 1.1 \% \quad \Theta_{B} \approx 19^{\circ} \\
& p_{V} \approx 1.2 \% \quad \Theta_{V} \approx 19^{\circ} .
\end{aligned}
$$

Comparison of all the above mentioned data suggests that the polarimetric variability for this star is small. Note that the Welsh test shows small but rapid variability of the Stokes parameter $Q$ in the $V$ band on JD...9747. According to Shevchenko (1989) this object is more likely a Be star, similar to $\gamma$ Cas, than a young star. 


\subsubsection{6. $\mathrm{LkH} \alpha 218$}

According to earlier data the amplitude of photometric variability of this object is about $2^{\mathrm{m}}$ in the $V$ band. It shows a large degree of polarization $(p \approx 2 \%)$ on the first night of observations (JD...9746). However on the second measurement (JD. . . 9751) no polarization was detected at the $95 \%$ confidence level in the $V$ and $B$ bands, but the polarization in the $U$ band was significant $(\approx 2.5 \%)$. Thus our data allow us to conclude that the polarization in the $V$ band is variable and that there is probably a significant wavelength dependence of polarization. We found the PA in the $U$ band to be different from that in the $B$ and $V$ bands by about $35^{\circ}-55^{\circ}$. Thus, an intrinsic component of polarization is clearly present.

\subsubsection{Z CMa (HD 53179)}

This object is now classified as a member of the FU Ori class of variables. It has been investigated intensively during the past ten years (see Whitney et al. 1993 and references therein). Its photometric behaviour was summarized by Covino et al. (1984) and Kolotilov (1991). During 1987 the star underwent a small optical outburst, when the optical flux increased from $V \approx 9^{\mathrm{m}} \cdot 5, B \approx 10^{\mathrm{m}} \cdot 6, U \approx 11^{\mathrm{m}} \cdot 0$ to $V \approx 8^{\mathrm{m}} \cdot 7, B \approx 9^{\mathrm{m}} \cdot 6, U \approx 9^{\mathrm{m}} \cdot 7$; this event was accompanied by an increase of polarization up to $5 \%$ (Miroshnichenko \& Yudin 1993). Detailed discussions of this behaviour were given by Miroshnichenko \& Yudin (1993) and Whitney et al. (1993). More recently Miroshnichenko et al. (1993) found that the star was slightly variable in the visible range between 1987 and 1991, with a mean $V \approx 9^{\mathrm{m}} .8$ and polarization $\approx 1.5 \%-2 \%$. They have pointed out a further fading of the object in $1991-1993\left(V \approx 10^{\mathrm{m}} \cdot 3, B \approx\right.$ $11^{\mathrm{m}} .5, U \approx 12^{\mathrm{m}} \cdot 2$ ) and noted changes in the polarization $\left(p_{\mathrm{R}} \approx 0.5 \%\right)$. This value of polarization is in good agreement with the data obtained by Jain et al. (1995) for that period. The data obtained here show an increase in polarization of up to $1.5 \%$ in $U B V$. No variability was detected at the $95 \%$ confidence level during the three nights of our observations. The present measurements indicate a flat wavelength dependence of polarization. Mean values of polarization are:

$$
\begin{array}{ll}
p_{U} \approx 1.3 \% & \Theta_{U} \approx 132^{\circ} \\
p_{B} \approx 1.6 \% & \Theta_{B} \approx 146^{\circ} \\
p_{V} \approx 1.4 \% & \Theta_{V} \approx 147^{\circ} .
\end{array}
$$

No photometric variability was detected at the $0^{\mathrm{m}} \cdot 01$ level during our observations. Note that according to Teodorani et al. (1998) the $V$ magnitude for the star was $V \approx 10^{\mathrm{m}} .2$ on JD. . 9742 (i.e. few days before our observations). In addition, note that $\mathrm{Z} \mathrm{CMa}$ is a well-known binary (see for example Baier et al. (1985) and the observed PA of polarization $\left(\simeq 147^{\circ}\right)$ is close to the direction between the components of the system $\left(\simeq 122^{\circ}\right)$.

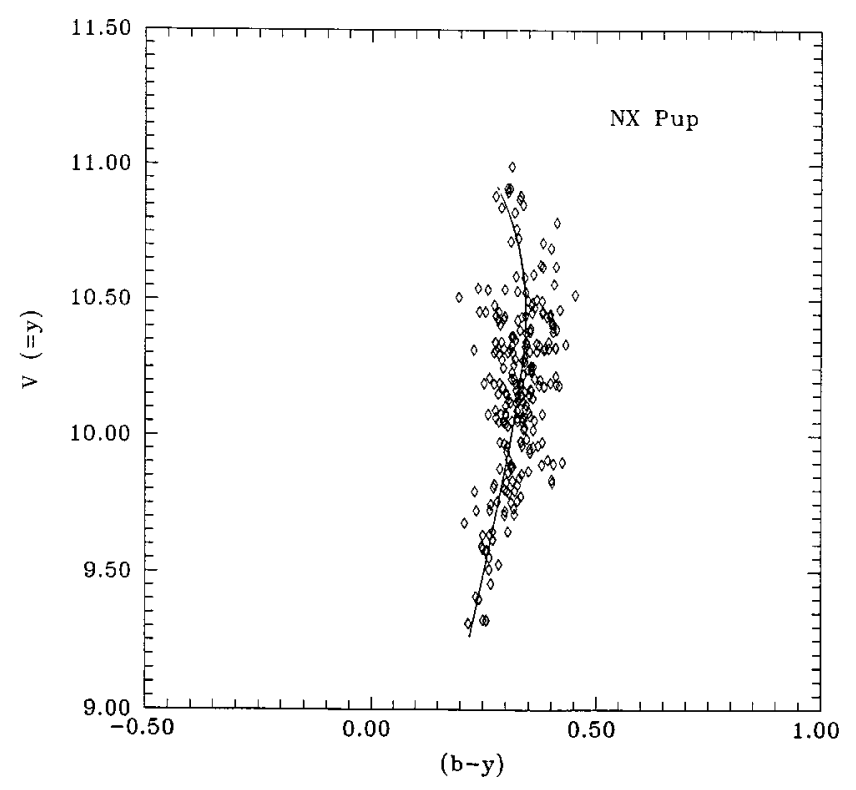

Fig. 4. Photometric behaviour of NX Pup

\subsubsection{NX Pup}

This object is a multiple system containing A7-F2, F5G8 and M0.5-M1.5 components (Tessier et al. 1994). According to Manfroid et al. (1991, 1994) and Sterken et al. $(1993,1995)$ it shows strong photometric variability from $V \approx 9^{\mathrm{m}} .3$ to $V \approx 11^{\mathrm{m}}$.0. No polarimetric measurements have previously been published. Our data indicate a small degree of polarization $(p \approx 0.9 \%)$ with $\mathrm{PA} \simeq 55^{\circ}$ at $V \approx 10^{\mathrm{m}}$. No polarization was detected at the $95 \%$ confidence level on JD. . 9742 in the $U B V$ bands but polarization at a statistically significant level was detected in the $V$ band on JD. . 9745. Nevertheless there is no variability in polarization at the $95 \%$ confidence level between the two sets of observations. Note that no photometric variability was detected in our measurements. It is interesting that the PA is close to that between the $\mathrm{A}$ and $\mathrm{B}$ components of the system $\left(\approx 62^{\circ}\right)$. More recently Scholler et al. (1996) have investigated this system in more detail and have estimated the spectral classes of the components as F0-F2 for NX Pup A and F7-G4 for NX Pup $\mathrm{B}$, with separation $125 \pm 3$ mas at PA $62^{\circ} 4 \pm 1$. 7 . They have estimated the interstellar extinction to the object as $A_{V}=0$ m.48 and deduced the presence of fast quasiperiodic oscillations in brightness with amplitude $\approx 0^{\mathrm{m}} \cdot 6$ and typical period $\approx 14^{\mathrm{d}}$. Both components were contained in the aperture used in our observations. Scholler et al. (1996) have pointed out that the IR excess of NX Pup A can be modelled by a viscous accretion disc at $\approx 20 \mathrm{AU}$ from the star, arising from interaction between the $\mathrm{A}$ and $\mathrm{B}$ components. While the $\mathrm{A}$ and $\mathrm{B}$ components are physically connected, the CS disc around NX Pup-A must be oriented in the direction of the $\mathrm{A}-\mathrm{B}$ components. Note that Blondel \& Tjin A Djie (1994), on the basis of low resolution IUE spectra, have pointed out that the disc 
around NX Pup is oriented "pole-on". This is consistent with the small polarization detected here, in contrast with the large IR excess. However, the main question to be answered in this case is the origin of the strong photometric variations of this object, mentioned above. More acceptable for NX Pup is a model with dust clouds revolving around the star as suggested for HAEBE stars with Algollike minima (Molster \& The 1994), but in terms of this model the disc around NX Pup must be oriented "edgeon". In this case the object should display low levels of polarization at high levels of brightness, as was detected here. Note that Scholler et al. (1996) have claimed that the general changes in colour with brightness for NX Pup can be explained by variable CS extinction. The photometric behaviour is shown in Fig. 4, and is similar to the behaviour detected for HAEBE stars with Algol-like minima. Scholler et al. (1996) proposed that the light seen from NX Pup near minimum brightness is mainly scattered light from a CS disc or envelope, while the direct light from the star is heavily obscured. The polarimetric data obtained here near maximum light suggest that the vector of observed polarization is approximately parallel to the disc plane, leading to the conclusion that there is an optically thick disc, or scattering in polar lobes above and below the disc. Additional polarimetric observations of the NX Pup system at minimum light and with better accuracy are needed to confirm this suggestion.

\subsubsection{RCW 34}

This object shows a high degree of polarization $(\approx 5 \%-6 \%)$ in all filters. Night-to-night variability at the $95 \%$ confidence level was not detected in any of the filters used. Also no photometric variability was detected in our measurements, at the level of 0 m.02. The average values of the degree of polarization and PA in the $V$ band are $5.6 \% \pm 0.2 \%$ and $59^{\circ} \pm 1^{\circ}$. Note however that the data of Jain et al. (1995) indicate a significantly smaller degree of polarization $(3.0 \% \pm 0.4 \%)$ in 1993 and a PA of about $79^{\circ} \pm 4^{\circ}$, significantly different from our data. Thus the object is strongly variable on a time-scale of years.

\subsubsection{He 3-331 (HD 85567)}

The polarization for this object does not exceed $0.5 \%$. Our five measurements on three nights show evidence of variability in both the degree of polarization and $\mathrm{PA}$ in the $V$ band in the sense that the degree of polarization on the last night was significantly smaller and the PA had changed by about $90^{\circ}$. The Welsh test suggests the presence of rapid variability of the Stokes parameters in the $V$ band at the $95 \%$ confidence level on JD. . .9744. In addition, night-to-night variability was also detected at the $95 \%$ confidence level. On JD. . 9751 polarization was

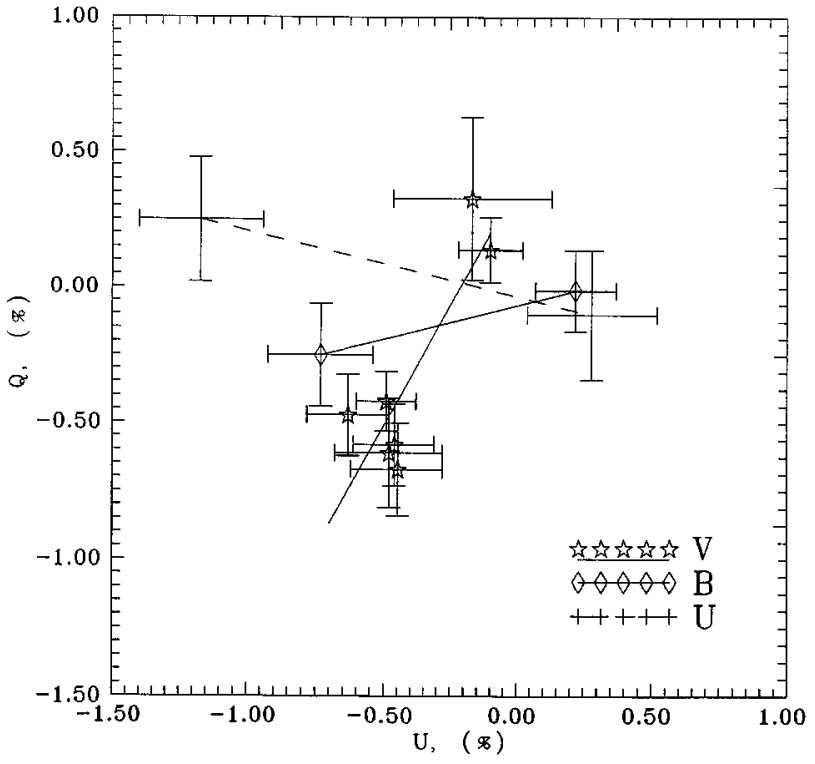

Fig. 5. $U-Q$ diagram for $\mathrm{He} 3-515$

statistically detected in the $B$ band only, and was absent in the $U$ and $V$ bands. The average value of polarization for the object is $p_{V} \approx 0.45 \%, \Theta_{V} \approx 107^{\circ}$. Note that no photometric variability was detected at the level of about $0 \mathrm{~m} .05$.

\subsubsection{He 3-515 (HD 94509)}

Strong night-to-night variability in both the degree of polarization and PA was detected. The object is possibly variable on a time-scale of minutes (see JD...947). The Clarke-Stewart test shows that no polarization was detected at the $95 \%$ confidence level in the $V$ band on JD. . 9749 and in the $U B V$ bands on JD. . . 9751, whereas on other dates the polarization was statistically significant. Note that the data points on the $U-Q$ plane are clustered along a line in each of the filters used but the orientation of these lines differ strongly from one another (see Fig. 5). In both sets of $U B V$ measurements it seems that the wavelength dependence of polarization is unlike that of interstellar polarization, the star showing increasing polarization at shorter wavelengths. However the relatively large errors do not allow us to draw definite conclusions.

\subsubsection{He 3-554 (HD 95881)}

This object shows variability of the Stokes parameters on a time-scale of minutes (see JD... 9755). The ClarkeStewart test shows the presence of polarization on all dates of observation. The variations of the Stokes parameter $U$ over 1 hour's monitoring on JD. . 9975 are shown in Fig. 6. The only $U B V$ measurement on JD. . 9749 indicates that the wavelength dependence of polarization is flat. All the 


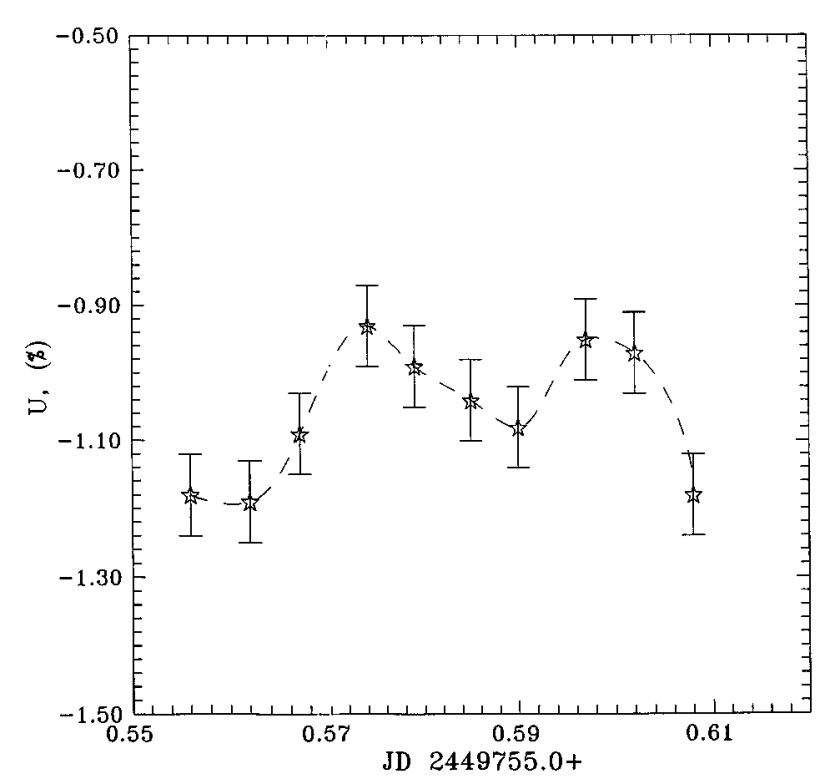

Fig. 6. Time variability of the Stokes parameter $U$ for He 3-554

above points to the important rôle of Thomson scattering in determining the polarization of this star.

\subsubsection{He 3-597 (HD 97048)}

This object showed no polarimetric variability (within the errors) during our observations. Besides the data obtained here are in good agreement with earlier results obtained by McGregor et al. (1994), Whittet et al. (1992) and Hutchinson et al. (1994). We present in Table 3 the polarimetric data from the above mentioned papers.

Taking into account the nonvariable polarization of the star and the similarity of the wavelength dependence of polarization to the interstellar polarization, there are two possibilities:

(a) there is no intrinsic polarization for this object and all observed polarization has an interstellar origin, or

(b) the intrinsic polarization is essentially stable.

In addition, note that Drissen et al. (1989), on the basis of their polarimetry at $\lambda=4300,5250,7500 \AA$ also classified this star as a nonvariable object, in agreement with Bastien (1985). Bastien (1985) has also pointed the similarity of the polarization of HD 97048 to interstellar, but noted a small $\left(\approx 5^{\circ}\right)$ rotation of $\mathrm{PA}$ as a function of wavelength. He has discussed this in terms of dichroic extinction by grains aligned in the reflection nebula, which is filamentary and has $\mathrm{PA} \approx 140^{\circ}$. Bastien (1985) also derived the interstellar $\mathrm{PA}$ in the vicinity of the star to be $\approx 118^{\circ}$. Note that neither our observations nor the more precise observations of Whittet et al. (1992), Hutchinson et al. (1994) and McGregor et al. (1994) show rotation of the PA in the optical. However it is important to note that the data obtained by Whittet et al. (1992) and McGregor

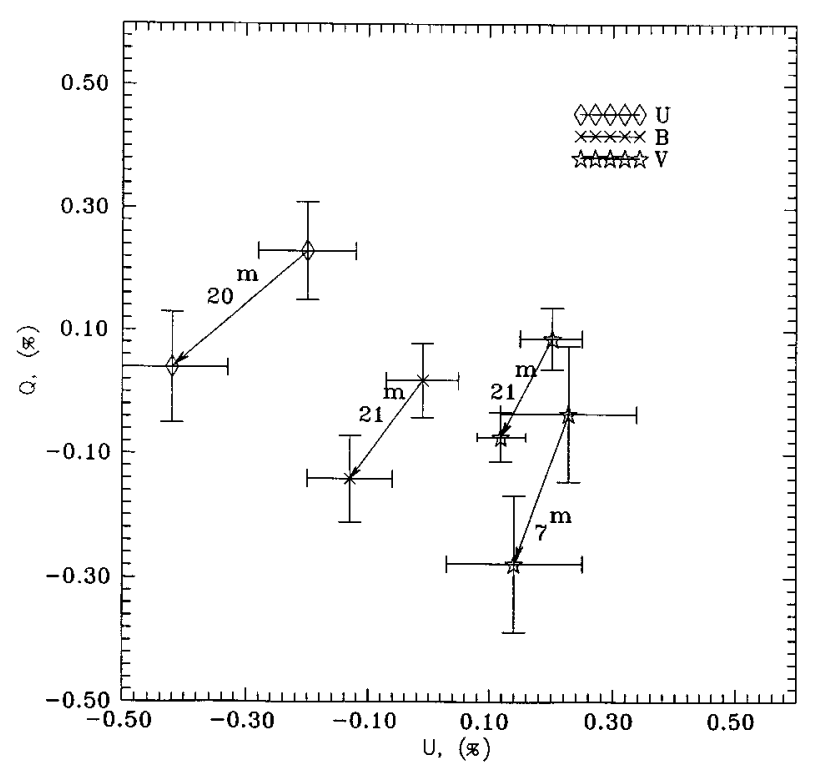

Fig. 7. $U-Q$ diagram for $\mathrm{He} 3-672$

et al. (1994) give clear evidence for rotation of the PA in the IR for $\lambda \gtrsim 1.6 \mu \mathrm{m}$, the PA in the $K$ band being $\approx 120^{\circ}$. Thus the interpretation of the observed polarization suggested by Bastien (1985) is confirmed.

\subsubsection{He 3-644 (HD 98922)}

Our measurements show small polarization at the level of about $0.3 \%$, with possible variability on a time-scale of days. No polarization was detected at the $95 \%$ confidence level on JD . . 9751 but on other dates the polarization was statistically detected. The degree of polarization increases to shorter wavelengths.

\subsubsection{He 3-672 (HD 100546)}

This object shows clear evidence of variability on a timescale of days and minutes. The data points on the $U-Q$ diagram for two measurements in different wavebands with a time separation of about 20 minutes is shown in Fig. 7. An additional measurement in the $V$ band for other nights with a time separation of about 7 minutes show the same behaviour. Note that the variation of the data points in each of the filters used on the $U-Q$ plane is statistically significant at the $95 \%$ confidence level and takes place along parallel lines. In two measurements on JD. . 9743 and JD. . .9748 the object shows an increase of polarization in the $U$ band with a dip in the $B$ band. Henning et al. (1994) have deduced the presence of a disclike configuration of the dust component around this object. 
Table 3. Previous polarimetric measurements of He 3-597. Ref. 1: Whittet et al. (1992); 2: Hutchinson et al. (1994); 3: McGregor et al. (1994)

\begin{tabular}{|c|c|c|c|c|c|c|c|c|c|c|}
\hline \multicolumn{2}{|c|}{$U$} & \multicolumn{2}{|c|}{$B$} & \multicolumn{2}{|c|}{$V$} & \multicolumn{2}{|c|}{$R$} & \multicolumn{2}{|r|}{$I$} & \multirow[t]{2}{*}{ Ref. } \\
\hline $\begin{array}{c}p \pm \sigma p \\
(\%)\end{array}$ & $\begin{array}{l}\Theta \pm \sigma \Theta \\
\text { (deg.) }\end{array}$ & $\begin{array}{c}p \pm \sigma p \\
(\%)\end{array}$ & $\begin{array}{l}\Theta \pm \sigma \Theta \\
\text { (deg.) }\end{array}$ & $\begin{array}{c}p \pm \sigma p \\
(\%)\end{array}$ & $\begin{array}{l}\Theta \pm \sigma \Theta \\
\text { (deg.) }\end{array}$ & $\begin{array}{c}p \pm \sigma p \\
(\%)\end{array}$ & $\begin{array}{l}\Theta \pm \sigma \Theta \\
\text { (deg.) }\end{array}$ & $\begin{array}{c}p \pm \sigma p \\
(\%)\end{array}$ & $\begin{array}{l}\Theta \pm \sigma \Theta \\
\text { (deg.) }\end{array}$ & \\
\hline $\begin{array}{l}2.01 \pm 0.14 \\
2.01 \pm 0.05\end{array}$ & $147.2 \pm 2.0$ & $\begin{array}{l}2.26 \pm 0.05 \\
2.28 \pm 0.02 \\
2.28 \pm 0.07\end{array}$ & $\begin{array}{l}141.0 \pm 1.0 \\
144.4 \pm 0.3\end{array}$ & $\begin{array}{l}2.57 \pm 0.08 \\
2.75 \pm 0.07 \\
2.65 \pm 0.07 \\
2.82 \pm 0.04\end{array}$ & $\begin{array}{l}143.0 \pm 1.0 \\
142.0 \pm 0.7 \\
145.0 \pm 0.8 \\
144.7 \pm 0.6\end{array}$ & $\begin{array}{l}2.76 \pm 0.06 \\
2.71 \pm 0.05 \\
2.66 \pm 0.04\end{array}$ & $\begin{array}{r}142.0 \pm 1.0 \\
143.7 \pm 0.5 \\
142.9 \pm 0.4\end{array}$ & $\begin{array}{l}2.53 \pm 0.05 \\
2.62 \pm 0.06 \\
2.59 \pm 0.08\end{array}$ & $\begin{array}{l}143.0 \pm 1.0 \\
143.5 \pm 0.7 \\
143.3 \pm 0.8\end{array}$ & $\begin{array}{l}1 \\
2 \\
2 \\
3\end{array}$ \\
\hline & $J$ & & $H$ & & $K$ & & & & & Ref. \\
\hline $\begin{array}{c}p \pm \sigma p \\
(\%)\end{array}$ & $\begin{array}{l}\Theta \pm \sigma \Theta \\
\text { (deg.) }\end{array}$ & $\begin{array}{c}p \pm \sigma p \\
(\%)\end{array}$ & $\begin{array}{l}\Theta \pm \sigma \Theta \\
\text { (deg.) }\end{array}$ & $p \underset{(\%)}{ \pm \pm \sigma p}$ & $\begin{array}{l}\Theta \pm \sigma \Theta \\
\text { (deg.) }\end{array}$ & & & & & \\
\hline $\begin{array}{l}1.32 \pm 0.13 \\
1.54 \pm 0.07\end{array}$ & $\begin{array}{l}144.0 \pm 2.6 \\
141.0 \pm 2.0\end{array}$ & $\begin{array}{l}0.42 \pm 0.03 \\
0.84 \pm 0.03\end{array}$ & $\begin{array}{l}129.2 \pm 1.5 \\
129.0 \pm 1.0\end{array}$ & $0.67 \pm 0$. & $05121 \pm 4$ & & & & & $\begin{array}{l}3 \\
1\end{array}$ \\
\hline
\end{tabular}

\subsubsection{He 3-692 (HD 101412)}

The degree of polarization for this object is less than $1 \%$. Nevertheless the object probably shows variability on a time-scale of minutes in all filters. On the $U-Q$ diagram the object shows similar behaviour to He 3-672 (see above) but relatively large errors do not allow us to draw definite conclusions. Note that this star does not show any signs of association with dark or reflection nebulae, and Eisloffel \& Ray (1994) have pointed out that it is inconsistent with the definition of HAEBE stars. No photometric variability was detected in our measurements at the level of 0 m.04.

\subsubsection{He 3-741 (HD 104247)}

This object is one of the brightest HAEBE stars and has a significant excess in the near IR. According to Blondel \& Tjin a Djie (1994) the distance is only $88 \mathrm{pc}$ and interstellar extinction is very small $\left(A_{V} \approx 0^{\mathrm{m}} \cdot 01-0^{\mathrm{m}} \cdot 02\right)$. Model calculations presented by Blondel \& Tjin a Djie (1994) indicate the presence of a disc, which is viewed at large inclination angle $\left(\approx 53^{\circ}\right)$. In our measurements the star shows a small degree of polarization $(\approx 0.3 \%)$. Note however that our data, which were separated by 6 days, indicate polarimetric variability: on JD. . .9745 polarization was certainly detected in the clear filter and in the $V$ band, whereas on JD. . 9751 no polarization at the $95 \%$ confidence level was detected in $U B V$.

\subsubsection{HD 132947}

The degree of polarization for this object is about $1 \%^{-}$ $1.5 \%$. Our measurements indicate variability on a timescale of days. It shows interesting behaviour on the $U-Q$ diagram: the data points for each filter are clustered along a line but the directions of these lines differ strongly from one another (see Fig. 8). This indicates that at least two components of polarization are present.

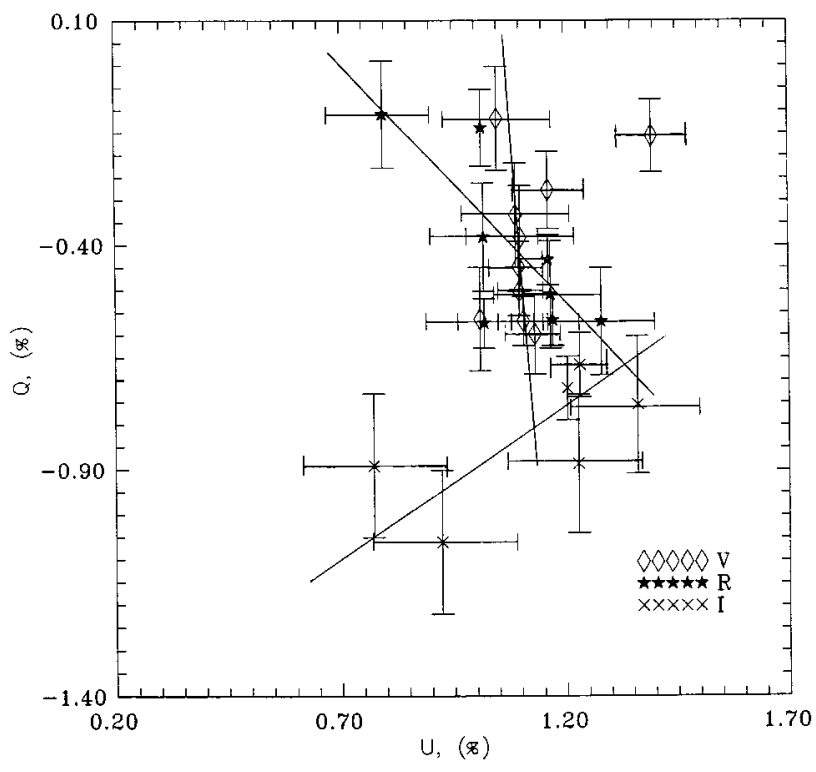

Fig. 8. $U-Q$ diagram for HD 132947

In addition, note that the degree of polarization shows a clear tendency to increase to the red. No photometric variability was detected on the level of about $0^{\mathrm{m}} \cdot 1$ in $V$, and the $V$ magnitude is in good agreement with the value in Table $1\left(\approx 8^{\mathrm{m}} \cdot 9\right)$.

\subsubsection{HD 141569}

According to Gahm et al. (1983) this star is a binary system with components $\mathrm{A} 0 \mathrm{~V}\left(V \approx 7^{\mathrm{m}} \cdot 1\right)$ and G0Vpec $(V \approx 12.7)$. The object shows polarization at a level of about $0.5 \%-0.9 \%$. No night-to-night variability at the $95 \%$ confidence level was detected in our three measurements. Some differences of polarization parameters in the $U$ band were detected on JD. . 9918 but the $\chi^{2}$-test shows strong deviations of the distribution of the Stokes parameters from the normal. Note however that the 
wavelength dependence of polarization is flat and unlike that of interstellar polarization. Thus an intrinsic component of polarization is indicated. No photometric variability was detected at the level of $0 \mathrm{~m} .1$ in $V$ and the photometry in $V$ was the same as noted in Table $1\left(\approx 7^{\mathrm{m}} \cdot 1\right)$.

\subsubsection{HD 142666}

An analysis of the spectral energy distribution for HD 142666, carried out by Waelkens et al. (1994), led them to conclude that the CS material around the star is distributed non-spherically and the inclination of the disc is high. Our only measurement in the $V R_{\mathrm{c}} I_{\mathrm{c}}$ bands shows a small degree of polarization, $\approx 0.7 \%$, with constant PA $\approx 76^{\circ}$. Nevertheless the wavelength dependence of polarization is unlike that of interstellar polarization and the degree of polarization probably increases to the red. Thus the polarization is intrinsic and is likely connected with Thomson scattering in the CS shell. Note that the visual magnitude in our single observation was about 8.7 , i.e. the star was observed at maximum light (see Table 1) whereas according to Bogaert \& Waelkens (1990) the amplitude of photometric variability of this star is about $3^{\mathrm{m}}$ in the $V$ band.

\subsubsection{RY Lup}

Polarization of this TTS was investigated intensively by Bastien (1985), Drissen et al. (1989) and Hutchinson et al. (1994). Our data also show variability of polarization. Further, the star shows largest polarization at minimum brightness. It is significant that this object is the only star from our survey which shows repeatedly circular polarization on a $3 \sigma$ level. A detailed discussion of the data will be given in a separate paper.

\subsubsection{MWC 863 (HD 150193)}

The evolutionary status of this object is not well established. It lies near the $\rho \mathrm{Oph}$ star forming region but is not associated with dark or reflection nebulae. We present in Table 4 previous polarimetric measurements.

Analysis of our data, together with those of Whittet et al. (1992) and Hutchinson et al. (1994), leads us to draw conclusions about the polarimetric variability of this star. Note that the data of Jain et al. (1995) are not in good agreement with those of other authors. This can be ascribed to differences in the filters used. On the $U-Q$ diagram in the $V R_{\mathrm{c}} I_{\mathrm{c}}$ bands we see clear evidence of an ellipse (see Figs. 9, 10). The same behaviour, but not so clearly, is found in the $U B$ bands. According to Reipurth \& Zinnecker (1993) and Jensen et al. (1996) MWC 863 is a binary system with angular separation of 1". 1 and projected component separation $150-176 \mathrm{AU}$. Reipurth \&

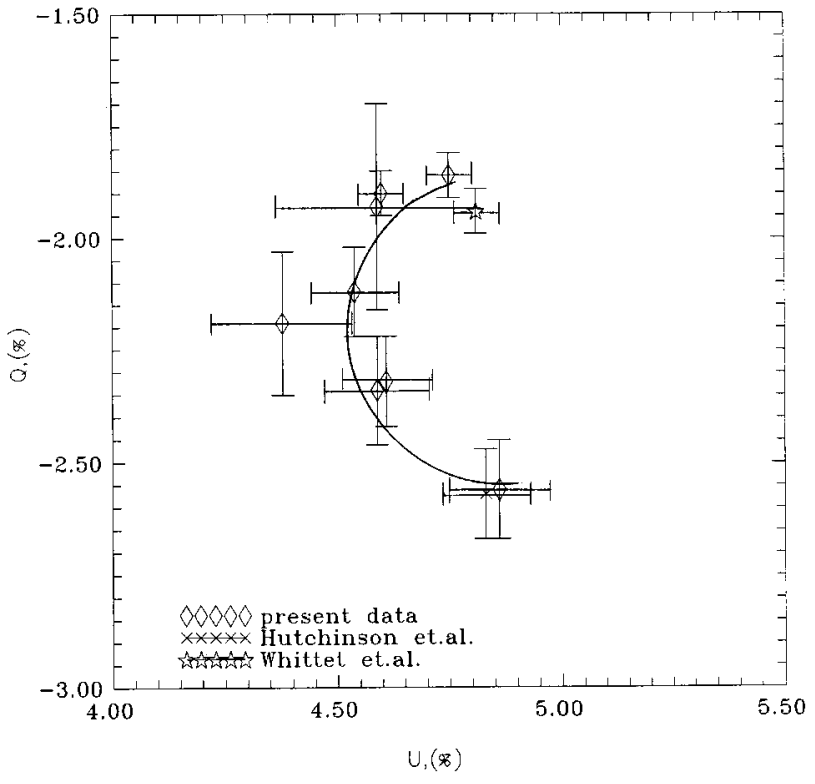

Fig. 9. $U-Q$ diagram $\left(R_{\mathrm{c}}\right.$ band) for MWC 863

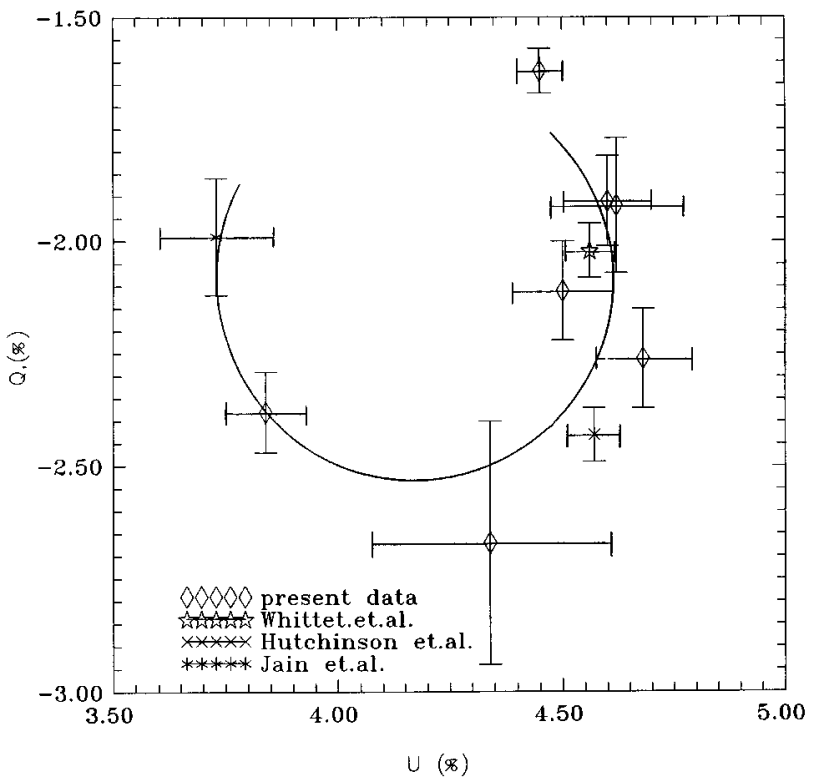

Fig. 10. $U-Q$ diagram ( $I_{\mathrm{c}}$ band) for MWC 863

Zinnecker (1993) have also noted that the PA between the components is $227^{\circ}$, close to the PA of observed polarization. To explain the variable extinction to the object Mitskevich (1995) suggested a model of the circumstellar shell with comet-like clouds which are revolving in eccentric orbits. It is interesting to note that the temporal variations of the Stokes parameters $(U$ and $Q)$ are anticorrelated in $U B$ and $V R_{\mathrm{c}} I_{\mathrm{c}}$ (see Fig. 11). This immediately suggests one of two possibilities, namely either the presence of different mechanisms of polarization in the UV and in the red, or there are contributions from the two stellar components in varying amounts. 
Table 4. Previous optical polarimetric measurements of MWC 863. Ref. 1: Whittet et al. (1992); 2: Hutchinson et al. (1994); 3: Jain et al. (1995); 4: Martin et al. (1992)

\begin{tabular}{|c|c|c|c|c|c|}
\hline$U$ & $B$ & $V$ & $R$ & $I$ & Ref. \\
\hline $\begin{array}{l}\Theta \pm \sigma \Theta \\
\text { (deg.) }\end{array}$ & $\begin{array}{cl}p \pm \sigma p & \Theta \pm \sigma \Theta \\
(\%) & \text { (deg.) }\end{array}$ & $\begin{array}{l}\Theta \pm \sigma \Theta \\
\text { (deg.) }\end{array}$ & $\begin{array}{ll}p \pm \sigma p & \Theta \pm \sigma \Theta \\
(\%) & \text { (deg.) }\end{array}$ & $\begin{array}{ll}p \pm \sigma p & \Theta \pm \sigma \Theta \\
(\%) & \text { (deg.) }\end{array}$ & \\
\hline $\begin{array}{l}3.19 \pm 0.1551 \pm 1 \\
3.98 \pm 0.2057 \pm 1\end{array}$ & $\begin{array}{l}4.32 \pm 0.0756 \pm 1 \\
4.54 \pm 0.0861 \pm 1 \\
5.32 \pm 2.0364 \pm 11 \\
4.23 \pm 0.3261 \pm 2 \\
4.78 \pm 1.1961 \pm 7\end{array}$ & $\begin{array}{l}5.08 \pm 0.0456 \pm 1 \\
5.37 \pm 0.0459 \pm 1 \\
4.61 \pm 1.3843 \pm 8 \\
4.89 \pm 0.1663 \pm 1 \\
4.88 \pm 0.1860 \pm 1 \\
5.12 \pm 0.1457 \pm 1\end{array}$ & $\begin{array}{l}5.19 \pm 0.0556 \pm 1 \\
5.48 \pm 0.1059 \pm 1 \\
4.52 \pm 0.2257 \pm 1 \\
4.75 \pm 0.0860 \pm 1 \\
4.91 \pm 0.1059 \pm 1\end{array}$ & $\begin{array}{l}4.99 \pm 0.0657 \pm 1 \\
5.18 \pm 0.0659 \pm 1 \\
3.39 \pm 0.5852 \pm 5 \\
4.23 \pm 0.1359 \pm 1 \\
4.64 \pm 0.1559 \pm 1\end{array}$ & $\begin{array}{l}1 \\
2 \\
3 \\
3 \\
3 \\
3\end{array}$ \\
\hline$J$ & $H$ & $K$ & $L$ & $M$ & Ref. \\
\hline $\begin{array}{cl}p \pm \sigma p & \Theta \pm \sigma \Theta \\
(\%) & \text { (deg.) }\end{array}$ & $\begin{array}{cl}p \pm \sigma p & \Theta \pm \sigma \Theta \\
(\%) & \text { (deg.) }\end{array}$ & $\begin{array}{cl}p \pm \sigma p & \Theta \pm \sigma \Theta \\
(\%) & \text { (deg.) }\end{array}$ & $\begin{array}{cl}p \pm \sigma p & \Theta \pm \sigma \Theta \\
(\%) & \text { (deg.) }\end{array}$ & $\begin{array}{cl}p \pm \sigma p & \Theta \pm \sigma \Theta \\
(\%) & \text { (deg.) }\end{array}$ & \\
\hline $3.27 \pm 0.0657 \pm 1$ & $2.26 \pm 0.0360 \pm 1$ & $1.68 \pm 0.0260 \pm 1$ & $1.7 \pm 0.171 \pm 17$ & $1.43 \pm 0.2173 \pm 4$ & 1,4 \\
\hline
\end{tabular}

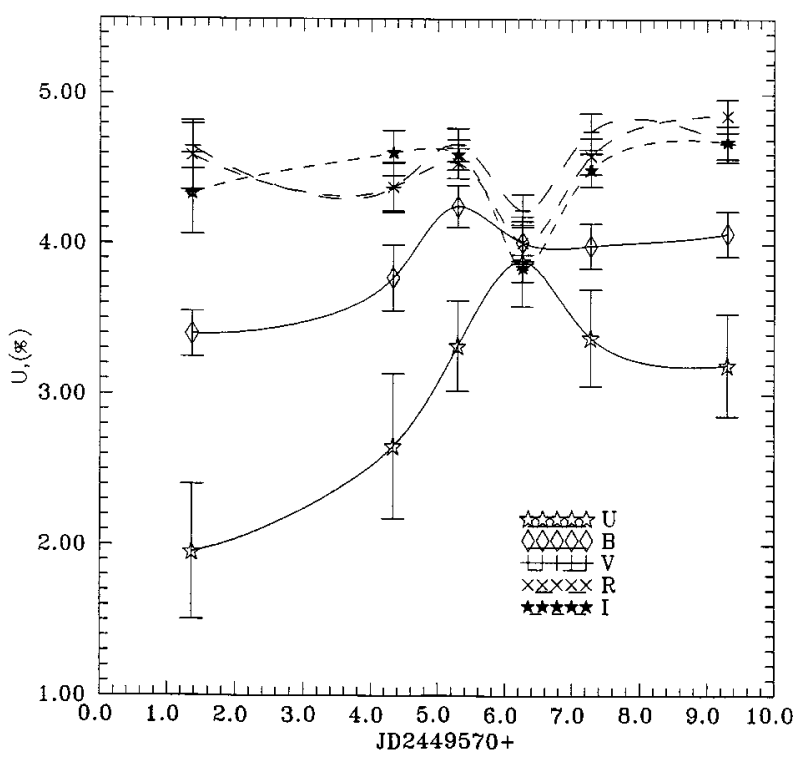

Fig. 11. Temporal variations of Stokes parameters $(U B$ and $V R_{\mathrm{c}} I_{\mathrm{c}}$ bands) for MWC 863

\subsubsection{KK Oph}

This object is a well-known binary with separation 2 '. 4 (Martin 1994). Grankin (1995) has pointed out that it shows evidence of periodic brightness variations with period $\approx 42^{\mathrm{d}}$ or $\approx 21^{\mathrm{d}}$ and amplitude up to $1^{\mathrm{m}}$. 0 . No photometric variability was detected in our observations at the level of about $0^{\mathrm{m}}$. 3 , and the star was observed at the midbrightness $V \approx 11^{\mathrm{m}} 4$. The Welsh test shows the presence of polarimetric variability at the $95 \%$ confidence level in all of the filters used, in three measurements on three consecutive nights. The wavelength dependence of polarization is completely different from interstellar, and was flat on JD. . 9575, JD. . . 9577; it was very peculiar on JD. . 9576

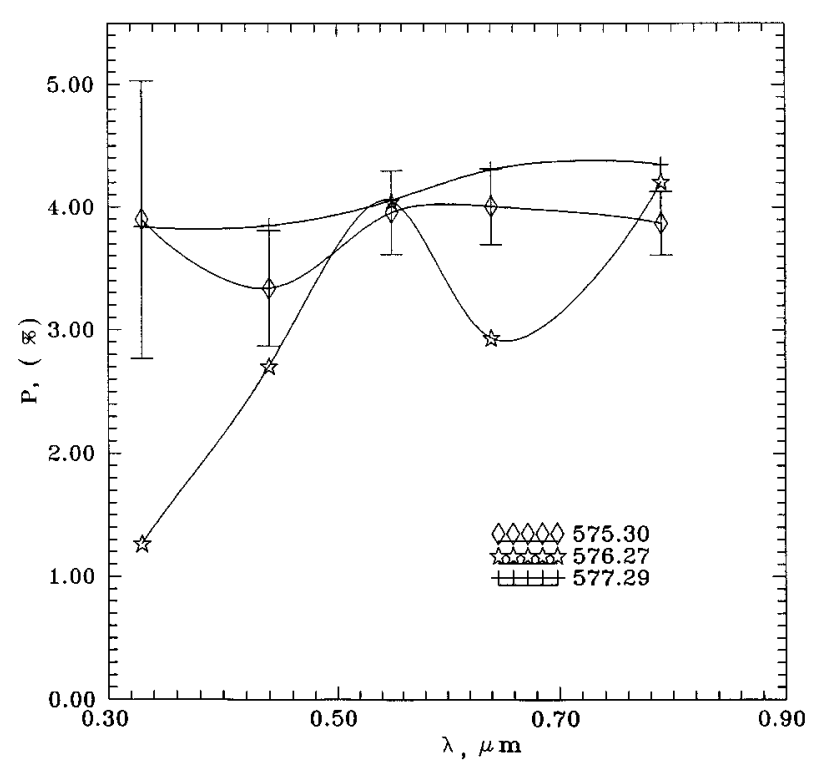

Fig. 12. Wavelength-dependence of polarization for KK Oph

(see Fig. 12). Unfortunately however, the large errors in the $U$ band polarization do not allow us to use these measurements to draw any conclusions about the wavelength dependence in detail.

Thus the polarization of this object has at least partly an intrinsic origin. In addition note that the observed PA $\left(\approx 170^{\circ}\right)$ is close to the direction between the components of the system $\left(\approx 340^{\circ}\right.$; Skinner et al. 1993).

\subsubsection{HD 163296}

An object of uncertain evolutionary status, HD 163296 was included in Allen \& Swings' (1976) list of B[e] stars, as well as in the list of Herbig Ae/Be stars (see Finkenzeller 
\& Mundt 1984; The et al. 1994). Catala (1994) has indicated that this object shows variability in some spectral lines on a very short time-scale (hours). He discussed this in terms of an origin of the variability at the stellar surface. Periodic modulation, with period $\approx 50^{\mathrm{h}}$, clearly emerges only in the $1989 \mathrm{Mg}$ II series. Henning et al. (1994) have interpreted the spectral energy distribution of the star in terms of an optically thin disc. Long wavelength emission from HD 163296 is probably dominated by a flared disc and/or an extended dust component (Mannings 1994). However this assumes that all the IRAS fluxes arise solely from a disc, which may not be the case. Polarimetric measurements were carried out by Barbier \& Swings (1982), Gnedin et al. (1992) and Jain et al. (1995). A brief discussion of the polarimetric behaviour was given by Gnedin et al. (1992), who pointed out that the polarization variations, with two months' separation, were unusual: the degree of polarization changed only in the red region, whereas the PA changed in the blue. Furthermore, their polarimetric data differed from that of Barbier \& Swings (1982) on two occasions in the degree of polarization and by $\approx 90^{\circ}$ in PA. They also demonstrated the presence of variability from night to night.

We obtained numerous polarimetric measurements and some of our results are as follows (see Beskrovnaya et al. 1998 for details):

(a) the only $U B V R_{\mathrm{c}} I_{\mathrm{c}}$ measurement shows that the maximum degree of polarization lies in the $U$ band, while in most of the $V R_{\mathrm{c}} I_{\mathrm{c}}$ measurements the degree of polarization increases to the red;

(b) the PA also changes from the $V$ to the $I_{\mathrm{c}}$ band;

(c) polarimetric variability was detected on a time-scale of days and hours. Fourier analysis shows the presence of two different periods in the polarization parameters with $P=7.5 \pm 0.3$ and $P=0.67$.

The variation with JD of nightly averages of the degree of polarization and PA are shown in Fig. 13.

The polarimetric behaviour of HD 163296 can be explained in terms of a model involving azimuthal inhomogeneities forming in different CS regions of a gaseous envelope, which are suspected to be magnetized. Detailed discussion of our data, together with spectral data, is given in a separate paper (Beskrovnaya et al. 1998).

\subsubsection{5. $\mathrm{LkH} \alpha 112$}

Our only $V R_{\mathrm{c}} I_{\mathrm{c}}$ measurement shows polarization for this object to be less than $0.5 \%$. The Clarke-Stewart test shows the presence of polarization at the $95 \%$ confidence level in the $V$ band only, and no polarization was detected in the $R_{\mathrm{c}}$ and $I_{\mathrm{c}}$ bands. Note that the brightness of the star was $\approx 9^{\mathrm{m}} .7$ in $V$, i.e. it was observed in the bright state (see Table 1).

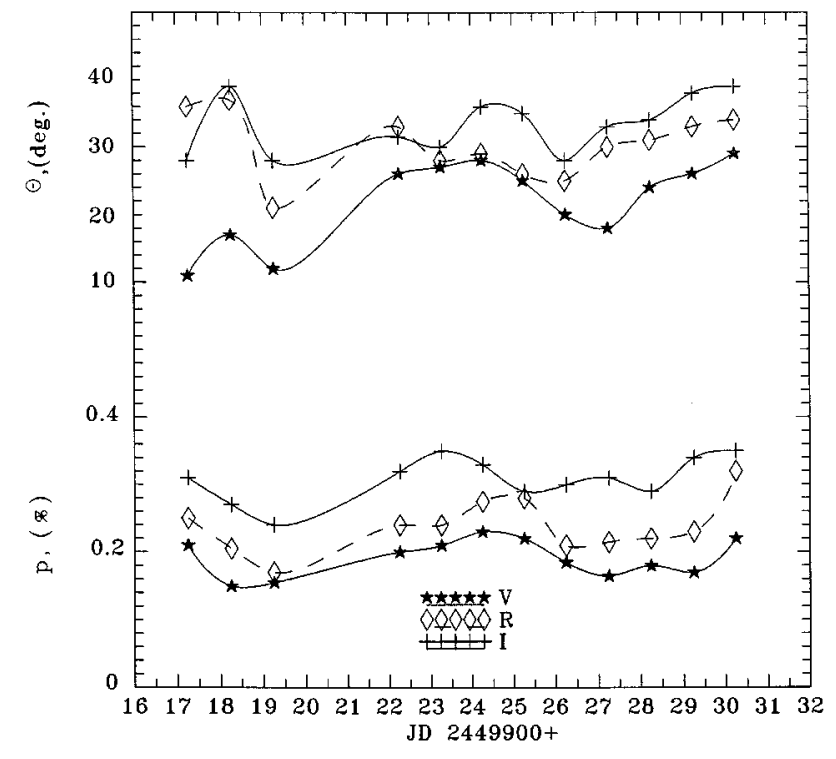

Fig. 13. Night-to-night variability of polarization degree and PA of HD 163296

\subsubsection{6. $\mathrm{LkH} \alpha 115$}

The degree of polarization of this object is high $(2 \%-$ $4 \%$ ). The Welsh test indicates the presence of polarimetric variability at the $95 \%$ confidence level on a time-scale of days. Probably there is a variation of PA from the ultraviolet to the red. Note that $\mathrm{LkH} \alpha 115$ is one of the HAEBE stars which showed strong circular polarization on two occasions at the $>4 \sigma$ level. However the $\chi^{2}$-test shows a strong deviation of the Stokes parameters from a normal distribution in the $V$ and $R$ bands on JD. . 9922 and JD. . 9926. Note that the star was observed close to minimum light $\left(V \approx 12^{\mathrm{m}} .1\right)$ and no photometric variability was detected at the level of $\approx 0$. 05 .

\subsubsection{VV Ser}

Our only measurement for this object shows polarization at the level of about $1 \%-2 \%$ with $V \approx 12^{\mathrm{m}}$. 0 .

\subsubsection{S CrA}

The polarization of this object was measured previously by Bastien (1985) and Hutchinson et al. (1994). Note that Hutchinson et al. (1994) found that the polarization of S CrA is variable both in degree and in PA on a time-scale of days. They also pointed out that a decrease in visual light was accompanied by a decrease in the degree of polarization. Such behaviour is unusual for HAEBE stars and was observed previously in only a few objects (e.g. Z CMa and MWC 137 above). Note however that according to Chelli et al. (1985) the object is a binary with PA between the components $\approx 147^{\circ}$ (separation of $1^{\prime \prime} .34$ and $\left.\Delta m=1^{m}\right)$, 


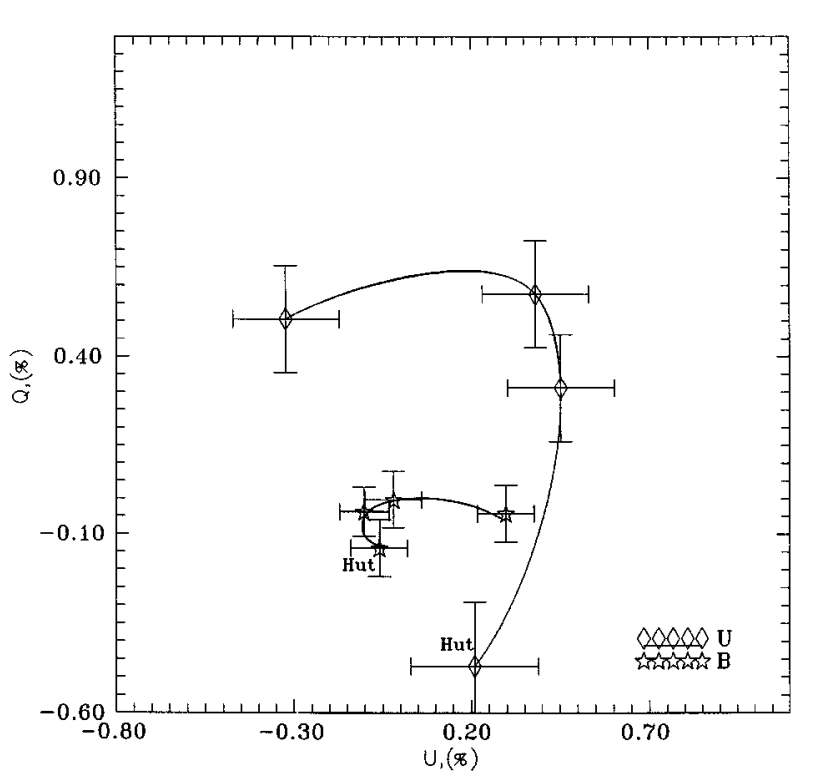

Fig. 14. $U-Q$ diagram for V1295 Aql

close to the PA of observed polarization. Our measurements on a single date of this object show polarization at the level of $\approx 1 \%-2 \%$, with increasing polarization to the violet; also there is some evidence for wavelengthdependence of PA. The visual magnitude of the object was $V \approx 11^{\mathrm{m}} .3$.

\subsubsection{V1295 Aql}

The polarization of this object is less than $1 \%$ in all of the filters. Note, however, that the object shows clear evidence of polarimetric variability at the $95 \%$ confidence level. Taking into account the data of Hutchinson et al. (1994) we can conclude that there are probably ellipse tracks in the $U B$ and $R_{\mathrm{c}}$ bands on the $U-Q$ diagram (see Fig. 14).

In addition, the wavelength dependence of polarization is also peculiar, namely, the degree of polarization has a maximum in the $U$ band in all our three measurements and shows a dip in the $B$ band (see Fig. 15).

The same behaviour was detected, but was not discussed, by Hutchinson et al. (1994). Note that, during our observations, the star had $V \approx 7^{\mathrm{m}} .9$ (i.e. close to the value presented in Table 1), with no evidence of variability at the level of about $0^{\mathrm{m}} .03$ in $V$. In addition, note that there is a statistically significant variation of $\mathrm{PA}$ of $\approx 90^{\circ}$ between the $U$ and $I$ bands.

\subsection{B[e] stars, extreme emission-line objects and other stars with IR excesses}

\subsubsection{HD 290770}

Our only measurement, in the $V$ band, shows negligible polarization for this object - less than $0.1 \%$. The Clarke-

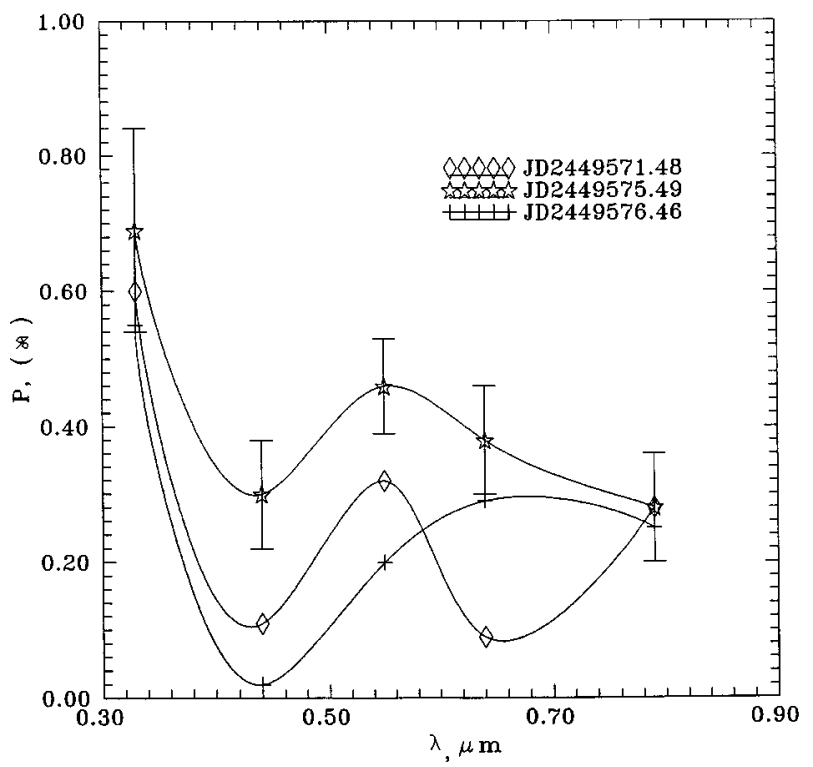

Fig. 15. Wavelength dependence of polarization of V1295 Aql

Stewart test shows that the detected polarization is not significant.

\subsubsection{AS 117}

The observed polarization of $\mathrm{AS} 117$ is about $0.5 \%$. However, the Clarke-Stewart test shows that polarization was detected at the $95 \%$ confidence level only on a single occasion in the $V$ band, on JD...9748. No polarization was statistically detected in the $U B V$ bands in our other observations. There is possible variation in the degree of polarization on a time-scale of days and variation of PA on a short time-scale (see JD. . . 9748). No photometric variability was detected at the 0.05 level in our measurements.

\subsubsection{AS 119}

The detected degree of polarization for this star is high $(\approx 2-4 \%)$. Note however, that polarization was statistically detected at the $95 \%$ confidence level only in the $V$ band in our measurements; it was not significantly detected in the $B$ and $U$ bands. The star shows evidence of polarimetric variability at the $95 \%$ confidence level in the $V$ band on a time-scale of days. The photometric data show that, during our observations, the object was fainter than $12^{\mathrm{m}}$ in the $V$ band, and probably the brightness of the star decreased by $\approx 0$. 15 on the last night of observations.

\subsubsection{MWC 158 (HD 50138)}

Grady et al. (1994) have pointed out that the available photometric and polarimetric data for a few $\mathrm{B}[\mathrm{e}]$ 
stars, including HD 50138, suggest a strong similarity to the known HAEBE stars viewed through their discs and displaying Algol-like minima. According to Bjorkman \& Schulte-Ladbeck (1994) (8 observations in 1990-1993) this $\mathrm{B}[\mathrm{e}]$ star shows large changes in $\mathrm{PA}$ and a possible loop in the $U-Q$ plane, which could be evidence for binarity or an orbiting dust cloud. According to Pogodin (1997) the envelope around the star is likely to be flattened close to the star. Our limited data do not allow us to draw any conclusions about the behaviour in the $U-Q$ plane but the Welsh test also indicates variability at the $95 \%$ confidence level in the $U$ and $V$ bands on a time-scale of days. Besides, our data differ from those obtained by Bjorkman \& Schulte-Ladbeck (1994), both in the degree of polarization and in PA. The wavelength dependence of polarization in the $U B V$ bands is likely flat or even shows increasing polarization in the $U$ band.

\subsubsection{EW CMa (27 CMa)}

Polarimetric observations for this star were previously reported by Serkowski (1970) and by Poeckert et al. (1979) and average values of polarization from these papers are, respectively, as follows:

$$
\begin{aligned}
& p_{U} \approx 1.10 \% p_{B} \approx 1.31 \% \quad p_{V} \approx 1.27 \% \\
& \Theta_{U B V} \approx 86^{\circ} \\
& p_{3400} \approx 0.60 \% \quad \Theta \quad \approx 91^{\circ} \\
& p_{4060} \approx 0.64 \% \quad \Theta \quad \approx 95^{\circ} .
\end{aligned}
$$

Thus the object is possibly variable, at least in the degree of polarization. Note however, that the observations of Poeckert et al. (1979) were carried out with narrow band filters. In our observation polarization was detected twice at the $95 \%$ confidence level in the $V$ band on JD...9748, and in the $U$ band on JD...9750. No polarization was detected in the $B V$ bands on JD. . 9750 and in $V$ on JD. . 9755. The Welsh test applied to our data, obtained with few days' separation, shows small variability of polarization in the $V$ band at the $95 \%$ confidence level. The average values of polarization are as follows:

$$
\begin{aligned}
& p_{U} \approx 0.4 \% \quad \Theta_{U} \approx 152^{\circ} \\
& p_{B} \approx 0.1 \% \quad \Theta_{B} \approx 140^{\circ} \text { (statistically not detected) } \\
& p_{V} \approx 0.1 \% \quad \Theta_{V} \approx 54^{\circ} .
\end{aligned}
$$

We note that the degree of polarization for this object was significantly less than that in previous measurements, indicating strong variability on a long time-scale. Probably there is a jump of $\mathrm{PA}$ of $\approx 90^{\circ}$ between the $U$ and $V$ bands. No photometric variability was detected at the 0 m.01 level in our measurements. All the above points to the presence of an intrinsic component of polarization. Note that, according to Balona (1995), the object shows variations of brightness with a period of about 1.262 .

\subsubsection{MWC 843 (HD 59319)}

This object shows variable polarization in both the degree of polarization $(\Delta p \approx 0.6 \%)$ and $\mathrm{PA}\left(\Delta \Theta \approx 40^{\circ}\right)$ on a time-scale of days. On JD. . 9745 and JD. . .9748 the polarization in the $V$ band was approximately the same. However, on JD. . 9752 the polarization in the $V$ band had changed. In the $B$ band variability was less but still significant at the $95 \%$ confidence level; no variation was found in the $U$ band.

\subsubsection{AS 160}

Polarization for this star was detected at the $95 \%$ confidence level in the $B$ and $V$ bands. It shows large and rapid variability in the $V$ band on a time-scale of days; furthermore, rapid variability on a time-scale of $\approx 10$ minutes was detected on JD. . 9744 at the $95 \%$ confidence level.

\subsubsection{Pup (HD 62623)}

According to Chelli et al. (1985), this star is a spectroscopic binary with a period of 137.767 . From previously published data (Manfroid et al. 1991, 1994, Sterken et al. $1993,1995)$ this star is a photometric variable in the $V$ band with amplitude $\approx 0$ m. 6 . Our observations were carried out on three nights and cover a period totalling 8 days. No photometric variability was detected on the above time-scale but the Welsh test shows the presence of small polarimetric variability at the $95 \%$ confidence level in the $V$ band on a time-scale of $\approx 0^{\mathrm{h}} .5$, as well as variability in the $U$ and $V$ bands on a time-scale of days. Average values of polarization for the star are as follows:

$$
\begin{array}{ll}
p_{U} \approx 1.37 \% & \Theta_{U} \approx 96.8 \\
p_{B} \approx 1.50 \% & \Theta_{B} \approx 96.3 \\
p_{V} \approx 1.48 \% & \Theta_{V} \approx 92.8 .
\end{array}
$$

\subsubsection{Hilt 641 (HD 64315)}

The only previous measurement of polarization for the object, obtained by Hiltner (1956) 30 years ago, shows polarization $p \approx 3.04 \%, \Theta \approx 114^{\circ}$ at $V \approx 9^{\mathrm{m}} \cdot 21,(B-V)=$ $0 \cdot 22,(U-B)=-0^{\mathrm{m}} \cdot 74$. Photometric data presented in the SIMBAD database indicate photometric variability for the $\operatorname{star}\left(9^{\mathrm{m}} \cdot 16<V<9^{\mathrm{m}} \cdot 43\right)$. We obtained numerous polarimetric data and can conclude that this object is a polarimetric variable. More detail of the polarimetric behaviour of Hilt 641 will be discussed in a separate paper. We note here however that the polarization of this star ranges between $3 \%$ and $3.5 \%$, and that there is a statistically significant variation of the PA between the $U$ and $V$ bands from $119^{\circ}$ to $115^{\circ}$.

\subsubsection{He $3-140$}

The degree of polarization for the object is about $1 \%$. Polarization was detected at the $95 \%$ confidence level in 
the $B$ and $V$ bands. We detected no polarimetric variability at the $95 \%$ confidence level. However there is probably a wavelength dependence of the $\mathrm{PA}\left(\approx 132^{\circ}, \approx 119^{\circ}\right.$, $\approx 94^{\circ}$ ) in the $U B V$ bands respectively.

\subsubsection{He $3-180$}

The degree of polarization for this object is about $2 \%$. It shows clear evidence of rapid polarimetric variability on a time-scale of $\approx 10$ minutes on both dates of observations. These variations were detected twice at the $95 \%$ confidence level. No photometric variability was detected at the level of 0.02 .

\subsubsection{He 3-365 (HD 87643)}

According to Shore et al. (1990), He 3-365 is a known variable $\left(\Delta V \approx 0^{\mathrm{m}} .5\right)$ and its CS shell is relatively dense. This object was included by The et al. (1994) in the list of extreme emission line objects and the full range of its brightness is $8^{\mathrm{m}} \cdot 51<V<9^{\mathrm{m}} \cdot 20$ (The et al. 1994; Manfroid et al. 1991, 1994; Sterken et al. 1993, 1995). According to McGregor et al. (1988) He 3-365 is a B[e] star for which a hot dust envelope is the defining characteristic, and has a $1.0-2.5 \mu \mathrm{m}$ continuum that rises to the red. They have pointed out that the star is surrounded by optical nebulosity and has not only a hot but also a cool dust shell. The commonly assumed extinction for the star is $A_{V} \approx 3^{\mathrm{m}}$. 1 . The optical-IR colour index $(V-L)_{\mathrm{obs}} \approx 6^{\mathrm{m}} \cdot 6$. Previous polarimetric measurements show a small degree of polarization $\left(p_{U} \approx 0.7 \%, p_{V} \approx 0.75 \%, p_{R} \approx 0.6 \%\right.$ with $\Theta \approx 133^{\circ} \pm 1^{\circ}-$ Barbier \& Swings (1982); $p_{V} \approx 0.6 \%$, $p_{R} \approx 0.55 \%$ with $\Theta \approx 133^{\circ} \pm 2^{\circ}-$ Gnedin et al. (1992). Our measurements show a larger level of polarization than reported previously, with an average degree of polarization $p_{U} \approx 1.2 \%, p_{B} \approx 1.1 \%, p_{V} \approx 0.8 \%$. The detected $\mathrm{PA}$ also differs, namely $15^{\circ}-20^{\circ}$ (the average value is about $\Theta \approx 155^{\circ}$ ). Note however that the observations of Gnedin et al. (1992) were carried out with a somewhat larger aperture $\left(27^{\prime \prime}\right)$ than ours. In contrast to the conclusion of Gnedin et al. (1992) the object is variable on a time-scale of days. In addition the object probably traces an ellipse in the $U-Q$ plane (see Fig. 16). More precise observations are needed in order to confirm this suggestion.

In addition we note that this object shows the same photometric behaviour as HAEBE stars with Algol-like minima. The star is bluer during brightness minima; see Fig. 17.

\subsubsection{WRA $15-535$}

This object was included by The et al. (1994) in the list of extreme emission line objects and its brightness was noted

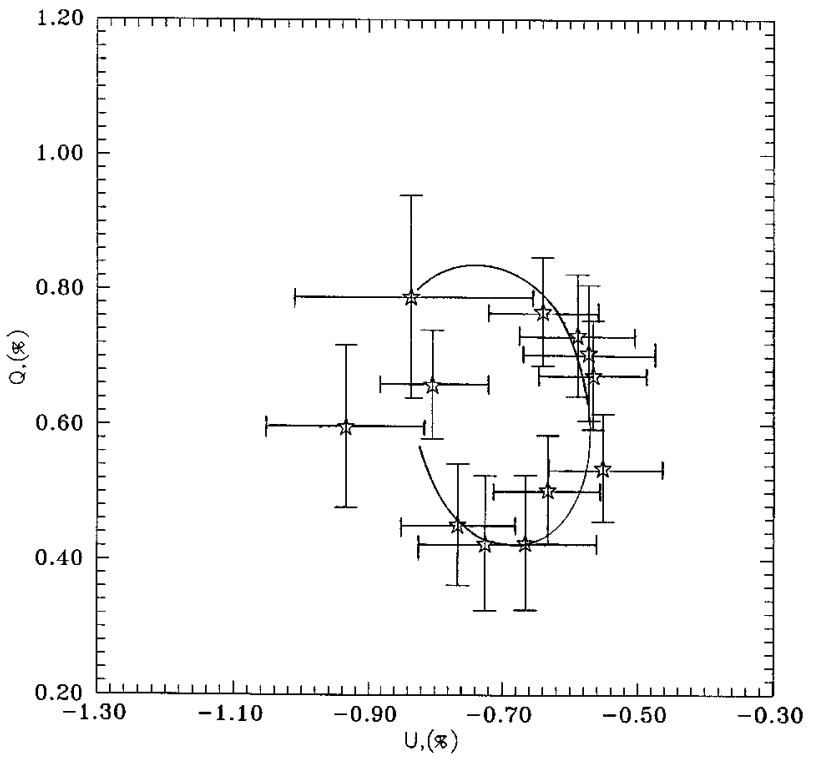

Fig. 16. $U-Q$ diagram for He 3-365

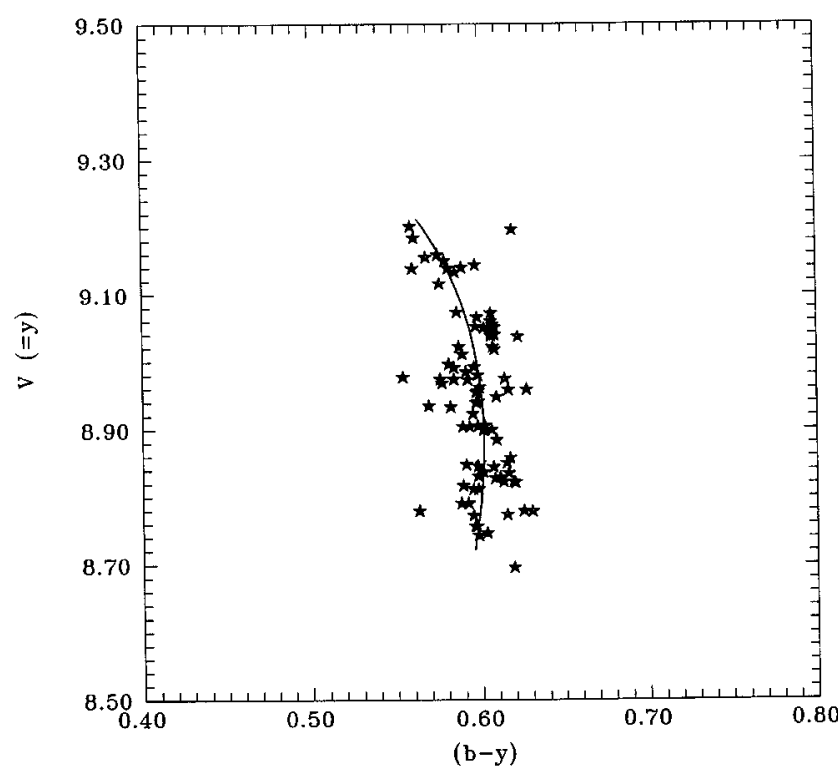

Fig. 17. Photometric behaviour of He 3-365

as 10.5 . However according to the data from the SIMBAD database it has $V=11^{\mathrm{m}} .4$. This object is a little-studied P Cygni star. According to McGregor et al. (1988) it has a $1.0-2.5 \mu \mathrm{m}$ continuum rising to the red, characteristic of thermal emission from dust. They also noted that their $K$ band photometry differs strongly from the value published by Allen \& Swings (1976), indicating IR variability. There is no evidence for the presence of optical nebulosity and cool dust around the star. The Welsh test shows that the object is a polarimetric variable in both the degree of polarization and PA, on a time-scale of days. On the $U-Q$ plane the star shows clear evidence of an elliptical track (see Fig. 18). 


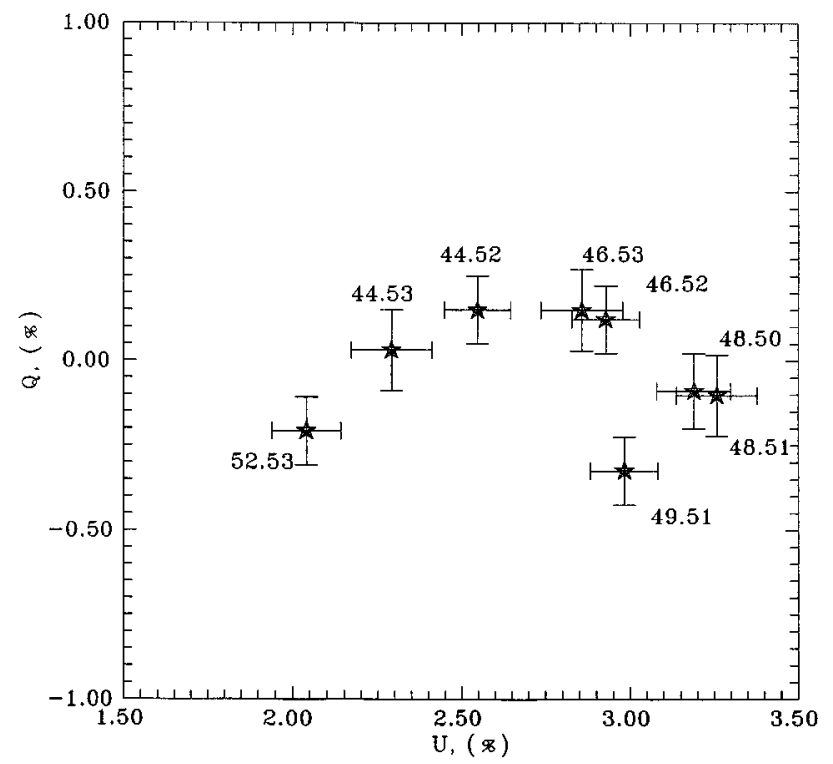

Fig. 18. $U-Q$ diagram for WRA 15-535

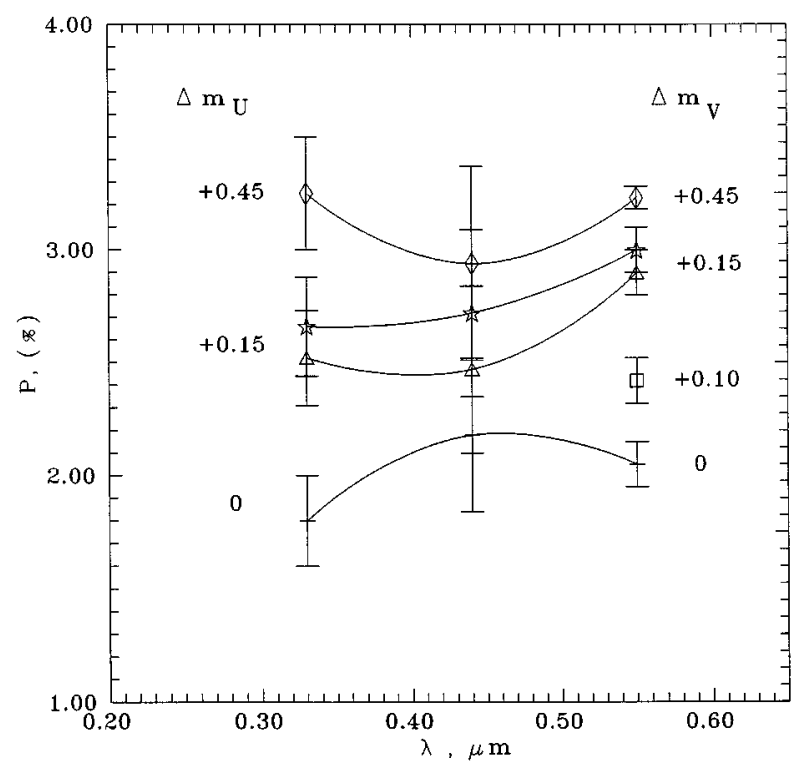

Fig. 19. Wavelength dependence of polarization and region of photometric variability of WRA 15-535

On the other hand there is a good correlation between the degree of polarization and the brightness of the star in the sense that it is brighter when the polarization is smaller, and vice versa (see Fig. 19); this is the same behaviour as that observed for HAEBE stars with Algol-like minima. Moreover, the wavelength dependence of polarization is also strongly variable, and depends on the level of brightness (see Fig. 19).

Note that the changes of brightness and polarization are in good agreement with that observed for HAEBE stars with Algol-like minima, in spite of the small amplitude. In addition note that according to Drilling (1991) this object is a supergiant of $\mathrm{OB}+\mathrm{Ie}, \mathrm{h}, \mathrm{i}$ spectral class. If this classification is correct the star is not young due to the large mass (see Palla \& Stahler 1990). Besides as will be shown in Sect. 4.1 the star also shows unusual far IR colours. Such interesting behaviour requires more detailed investigation.

\subsubsection{HD 90578}

This object shows high polarization. Average values of polarization are as follows:

$$
\begin{array}{ll}
p_{U} \approx 2.5 \% & \Theta_{U} \approx 124^{\circ} \\
p_{B} \approx 2.3 \% & \Theta_{B} \approx 129^{\circ} \\
p_{V} \approx 2.3 \% & \Theta_{V} \approx 123^{\circ} .
\end{array}
$$

Small polarimetric variability was statistically detected at the $95 \%$ confidence level in the $V$ band on a time-scale of days. Note that the wavelength dependence of polarization in $U B V$ is flat.

\subsubsection{HD 303004}

The variability range of HD 303004 in the visual is $10^{\mathrm{m}} \cdot 0<$ $V<11^{\mathrm{m}} .8$. Our polarimetric measurements were made when the brightness of the object was about $V \approx 10^{\mathrm{m}}$. No photometric variability was detected on the 0.02 level in our measurements. This object shows a high degree of polarization $(\approx 4 \%)$ in all of the filters. However, no polarimetric variability was detected at the $95 \%$ confidence level in any of the filters used. Average values of polarization are as follows:

$$
\begin{array}{ll}
p_{U} \approx 4.1 \% & \Theta_{U} \approx 112^{\circ} \\
p_{B} \approx 4.3 \% & \Theta_{B} \approx 116^{\circ} \\
p_{V} \approx 4.3 \% & \Theta_{V} \approx 113^{\circ} .
\end{array}
$$

It would be interesting to measure the polarization at minimum light.

\subsubsection{WRA $15-689$}

We made two measurements separated by six days for this object; its polarization is nonvariable at the $95 \%$ confidence level. Average values of polarization are as follows: $\begin{array}{ll}p_{U} \approx 1.4 \% & \Theta_{U} \approx 177^{\circ} \\ p_{B} \approx 0.9 \% & \Theta_{B} \approx 176^{\circ} \\ p_{V} \approx 1.0 \% & \Theta_{V} \approx 16^{\circ} .\end{array}$

There is probably a variation of the PA from $U$ to $V$ (by $\left.\approx 20^{\circ}\right)$. This fact indicates the presence of an intrinsic component of polarization. However the errors in the PA are not small. No photometric variability was detected at the level of 0 m.05 in our measurements.

\subsubsection{MWC 1264 (HD 152291)}

Our only $V R_{\mathrm{c}} I_{\mathrm{c}}$ measurement for this object indicates polarization at the level of about $1 \%$. Note the increasing degree of polarization from the visual to the red. 


\subsubsection{MWC 257 (HD 156702)}

In our three measurements, obtained over a time interval of a week, this star did not show polarimetric variability in the $U$ and $B$ bands; however in the $V R_{\mathrm{c}} I_{\mathrm{c}}$ bands variability was statistically detected at the $95 \%$ confidence level. The spectral distribution of the degree of polarization has a maximum in the $B$ band, decreasing to the red. No photometric variability was detected in our measurements at the level of $0^{\mathrm{m}} .1$ and the brightness of the star was $V \approx 8^{\mathrm{m}} \cdot 75$.

\subsubsection{MWC 272 (HD 316285)}

According to Lopes et al. (1992) the star is "probably one of the most luminous objects in the P Cygni class of stars". Lopes et al. (1992) have derived the spectral class of the object as about B0I, or even earlier, with $M_{*}=60 M_{\odot}$. McGregor et al. (1988) have pointed out that the star has a $1.0-2.5 \mu \mathrm{m}$ continuum which is essentially flat, and suggested that this is due to free-free emission. They have also noted that HD 316285 shows no hot dust emission, but shows strong far IR emission from cool dust. This object was included by The et al. (1994) in the list of extreme emission line objects but later, van der Veen et al. (1994) classified it as a member of the LBV class. They estimated the interstellar extinction to be about $A_{V} \approx 5^{\mathrm{m}}$. Taking into account a large mass of the star it is probably not a young. According to our data the polarization is about $3 \%$. In our two measurements, separated by a time interval of about a year, the star shows no variability in degree of polarization but the PA probably changed by about $5^{\circ}$, from $\approx 166^{\circ}$ in 1994 to $161^{\circ}$ in 1995 . Note that the data of Hutchinson et al. (1994), obtained with the same instrument and aperture in 1987, show a small variation in the degree of polarization in the $R_{\mathrm{c}}$ and $I_{\mathrm{c}}$ bands $(\approx 0.2 \%)$ but the PA measured at that time was $\approx 158^{\circ}$. The variations of the PA are significantly greater than the errors. The variability of polarization in the $U-Q$ plane is shown in Fig. 20. The Welsh test suggests clear evidence for variability in the Stokes parameters in all filters used at the $99 \%$ confidence level, on a time-scale of a year. As can be seen in Fig. 20 the data points in $U B V R_{\mathrm{c}} I_{\mathrm{c}}$ are clustered along a line in 1994. In 1995 the data points in $V R_{\mathrm{c}} I_{\mathrm{c}}$ are also concentrated along a line, but at a different angle. The same behaviour is also observed in the $B V R_{\mathrm{c}}$ data obtained by Hutchinson et al. (1994) in 1987, which is also at a different angle, but their data for the $I_{\mathrm{c}}$ band are peculiar.

From our data, together with those of Hutchinson et al. (1994), we can conclude that the wavelength dependence of polarization for HD 316285 is flat in the $B V R_{\mathrm{c}} I_{\mathrm{c}}$ bands, with decreasing of degree of polarization in the $U$ band (see Fig. 21); this behaviour is very similar to that of the of "classical" Be stars (see for example the Fig. 3 of Poeckert et al. (1979).

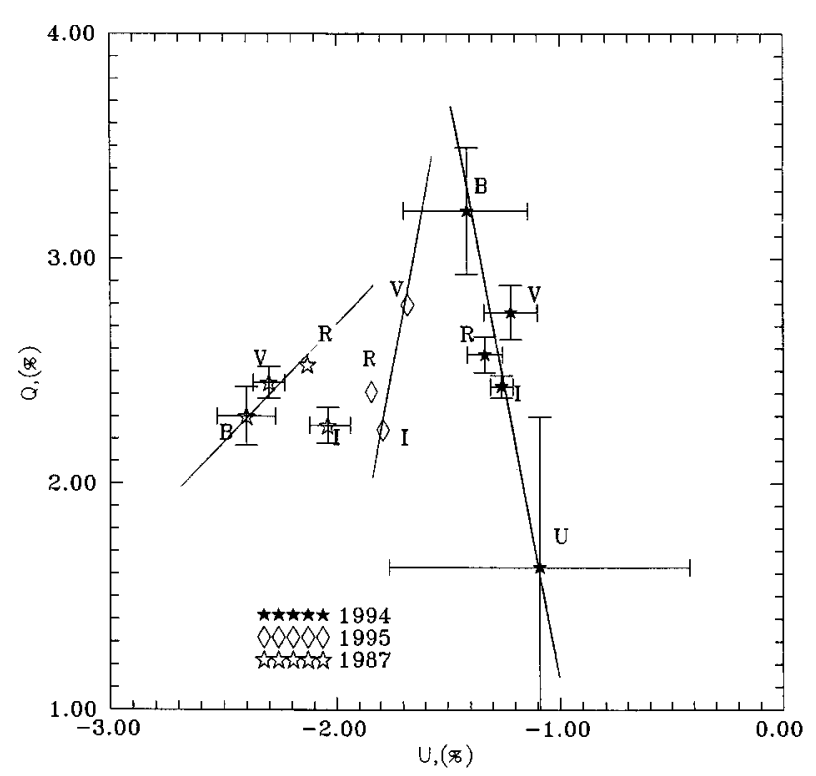

Fig. 20. $U-Q$ diagram for HD 316285 . Error bars smaller than plotted points unless overwise indicated

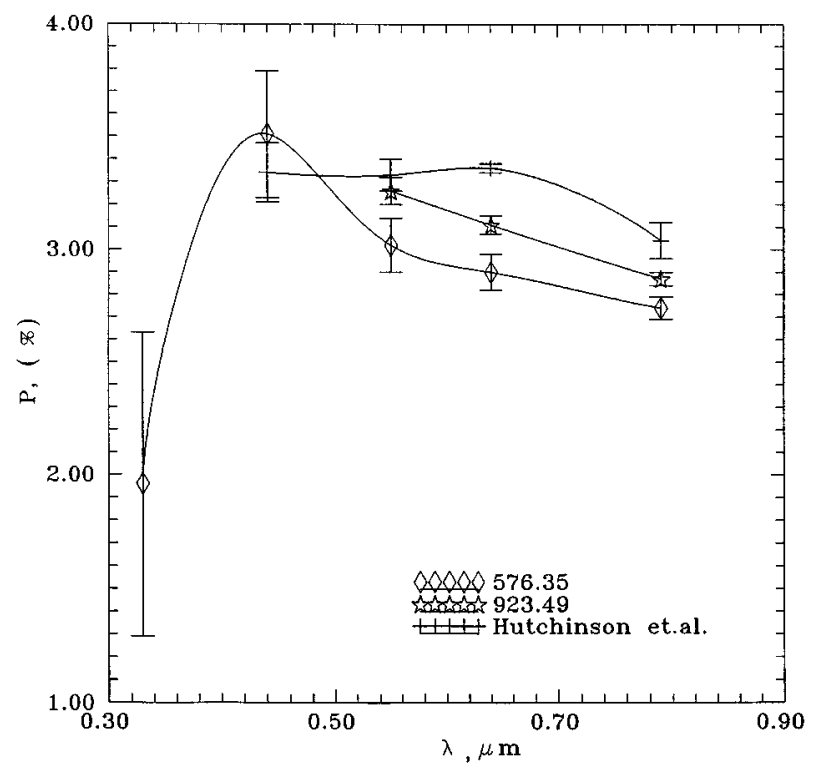

Fig. 21. Wavelength dependence of polarization of HD 316285

Such behaviour can easily be explained by supposing that Thomson scattering is the main mechanism of polarization for this object. This conclusion on the important rôle of Thomson scattering is consistent with the results of McGregor et al. (1988), mentioned above. In addition we note that, in 1994 , the star had $V \approx 9^{\mathrm{m}} .1$, whereas in 1995 the star was brighter in $V$ by at least $0^{\mathrm{m}}$. 1 .

\subsubsection{MWC 900 (HD 164246)}

On the basis of our measurements this object showed no polarimetric variations at the $95 \%$ confidence level, with average values of: 


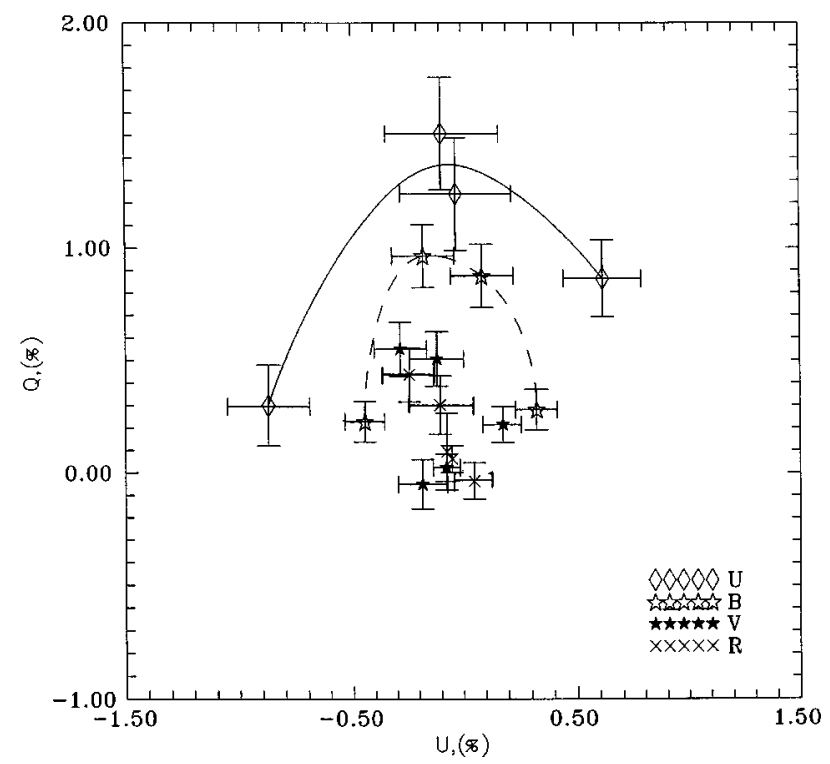

Fig. 22. $U-Q$ diagram for MWC 925

$$
\begin{aligned}
& p_{V} \approx 0.26 \% \quad \Theta_{V} \approx 176^{\circ} \\
& p_{R_{\mathrm{c}}} \approx 0.46 \% \quad \Theta_{R_{\mathrm{c}}} \approx 165^{\circ} \\
& p_{I_{\mathrm{c}}} \approx 0.30 \% \quad \Theta_{I_{\mathrm{c}}} \approx 151^{\circ} .
\end{aligned}
$$

There may be a variation in PA from the $V$ to the $I_{\mathrm{c}}$ band.

\subsubsection{MWC 925 (HD 169142)}

This object shows polarization at about the $1 \%$ level or less. However it shows clear evidence of polarimetric variability at the $95 \%$ confidence level on a time-scale of days in the $U B V$ bands. The polarization in the $R_{\mathrm{c}}$ band was statistically detected only on a single occasion, on JD. . .9574. No statistically significant polarization was detected in the $I_{\mathrm{c}}$ band in any of our measurements. On the $U-Q$ diagram we see evidence of an ellipse in all of the filters used (see Fig. 22).

The remarkable wavelength dependence of polarization for this object is shown in Fig. 23.

Note that the wavelength dependence is not like that of interstellar polarization, and the star shows strongly increasing polarization to shorter wavelengths. The sharp rise of the wavelength dependence is evidence for Rayleigh scattering by small grains in the CS envelope of MWC 925. Oudmaijer et al. (1992) have classified this star as Be and as a binary. It is an important point that MWC 925 was considered earlier to be a protoplanetary nebula or a star in the AGB/post AGB star to planetary nebula transition (Kaufl et al. 1993; van der Veen et al. 1989).

\section{Statistical study of the polarimetric properties}

\subsection{Evolutionary status of the programme stars}

To carry out a statistical study of the polarimetric properties of the stars in our survey more detailed selection

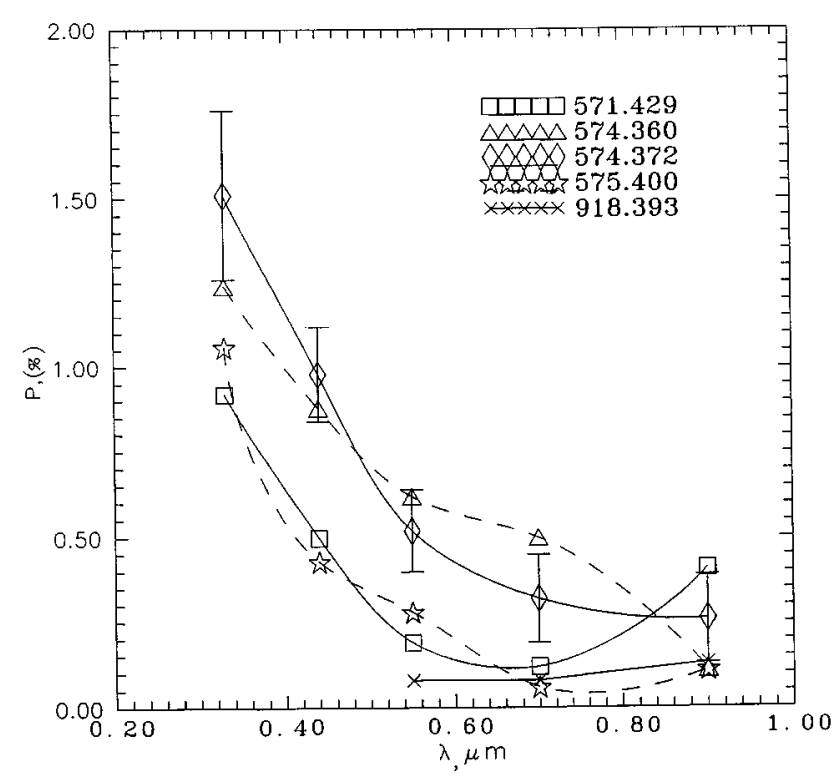

Fig. 23. Wavelength dependence of polarization of MWC 925

of the programme stars on the basis of their evolutionary status should be made. At present no definite criteria exist to distinguish peculiar early type stars (HAEBE stars, B $[\mathrm{e}]$, Be stars etc.) at different stages of evolution, and lists of these stars in the literature are inhomogeneous and partially overlap. One means of aiding classification is the location of objects in the IRAS two-colour $\left(\log \left[F_{25} / F_{12}\right]-\log \left[F_{60} / F_{25}\right]\right)$ diagram. It is well known that different stellar types (TTS, OH/IR stars, etc.) have a definite position in the IRAS two-colour diagram (see e.g. Weintraub 1992 and references therein; Harris et al. (1988); Odenwald 1989; Campbell et al. 1989; Strom et al. 1989 etc.). In addition such a comparison for different types of young stellar objects (YSO) was made by Yudin (1990), who showed that there is a certain sequence in the CS shell evolution of YSOs in the IRAS two-colour diagram, as follows:

(a) IR sources in star forming regions;

(b) IR sources with evidence of matter outflow and/or with the properties of TTS;

(c) "active" TTS and HAEBE stars with evidence of matter outflow;

(d) "classical" TTS and HAEBE stars;

(e) stars close to main sequence and/or "young" main sequence stars.

At present there is general consensus that those preMS stars which have the greatest IR excess are presumably the youngest. On the other hand, outflow activity is also believed to characterize sources at the earliest stages of evolution (see Tamura \& Sato 1989; Berrilli et al. 1989). According to Tamura \& Sato (1989), on the $\log \left[F_{25} / F_{12}\right]-\log \left[F_{60} / F_{25}\right]$ diagram, "more active" TTSs/YSOs are located on the cooler/redder side of the area defined by TTSs. Note that the above mentioned 


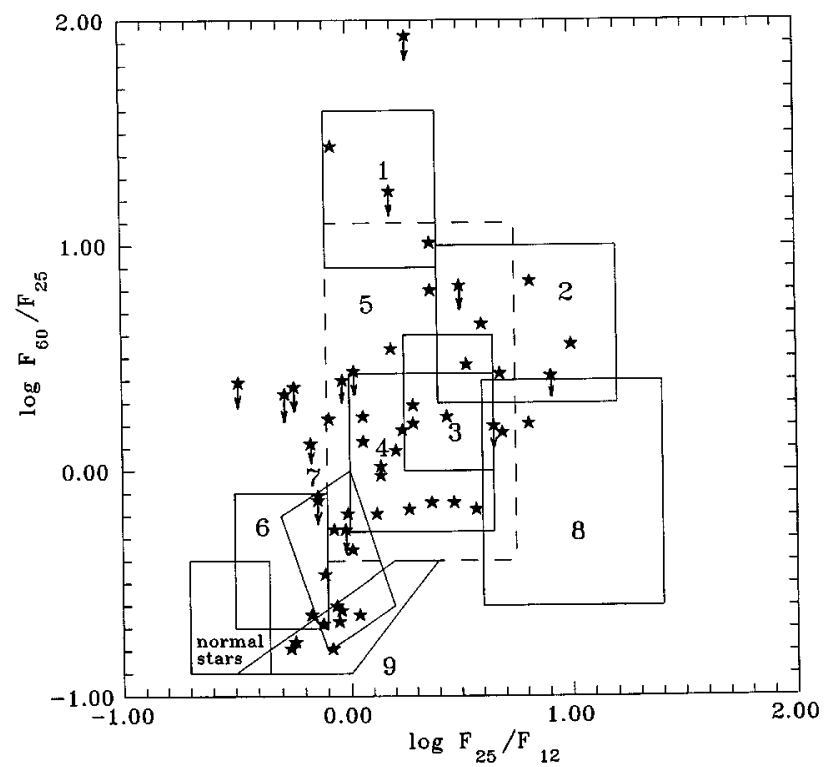

Fig. 24. IRAS two-colour diagram for objects at different stages of evolution. The box numbers correspond to the following objects: 1: IR sources in star forming regions; 2: IR sources with matter outflows; 3: IR sources with the properties of TTS; 4: TTS; 5: HAEBE stars: 6: classical Be stars; 7: peculiar B[e] stars; 8: OHPN and PN; 9: OH/IR stars

evolutionary sequence is in agreement with that discussed by Tamura \& Sato (1989) for low-mass young stellar objects:

(a) the objects on the earliest stages of evolution with extreme mass-outflow phenomena;

(b) the objects at the earliest $\mathrm{T}$ Tauri phase - which are older than the objects above but still associated with outflow phenomena;

(c) the "normal" TTS;

Tamura \& Sato (1989) have discussed the changes of appearance in IR polarization in terms of evolutionary changes in the optical thickness of the disks and bipolar reflection nebulae. Thus, the positions of stars on the IRAS two-colour diagram are reflected in their spectral energy distributions or, put another way, the positions of stars on the IRAS diagram, in the simple case, reflect the perturbation of dust in their CS environment. Note that, according to Weintraub (1992), HAEBE stars are located in a very large area ranging approximately between $-0.1<$ $\log \left[F_{25} / F_{12}\right]<0.75$ and $-0.4<\log \left[F_{60} / F_{25}\right]<1.05$. We should try to construct such a diagram using the IRAS data for $\mathrm{Be}$ and $\mathrm{B}[\mathrm{e}]$ stars and other early type objects (see Fig. 24).

Cote \& Waters (1987) have shown that classical Be stars occupy a small area of the IRAS colour-colour diagram that is consistent with that expected for free-free emission from a star surrounded by ionized gas and no dust. The far IR colours of most of Be stars are very similar, i.e. independent of spectral type (from B0 to B8) or luminosity class. This small area in the diagram is in the range $-0.50<\log \left[F_{25} / F_{12}\right]<-0.1$ (for $\approx 100 \%$ of 59 stars) and $-0.7<\log \left[F_{60} / F_{25}\right]<-0.1$ (for $\approx 80 \%$ of 19 stars). It is interesting that most $\mathrm{B}[\mathrm{e}]$ stars are also located in the same region, in a position that is close to that of main sequence stars. It is well known that peculiar $\mathrm{B}[\mathrm{e}]$ stars are characterized by the presence of hot $(\approx 1000 \mathrm{~K})$ CS dust, but as they have no cool dust there is no effect on their far IR excess; there is therefore a small but significant difference between the locations of Be and B[e] stars on the IRAS two-colour diagram in that the latter mainly have $\log \left[F_{60} / F_{25}\right]<-0.4$. A further important factor is that some $\mathrm{B}[\mathrm{e}]$ stars were suggested to represent transition stages or early evolutionary stages of planetary nebulae. Taking into account the above approach we have plotted the stars from our survey on the IRAS two-colour diagram to select objects according to their evolutionary status. The IRAS colours for the programme stars are given in Table 5 .

It is easy to see that the stars V1295 Aql, He 3-644, He 3-140, He 3-331, AS 119, MWC 158, He 3-554, AS 160, 3 Pup, and MWC 900 are located in the lower part of the boxes occupied by Be and $\mathrm{B}[\mathrm{e}]$ stars, far from the region occupied by HAEBE stars and close to the position occupied by normal stars. We conclude that they are definitely not young!

The stars He 3-515, He 3-692, He 3-741, KK Oph, MWC 863 and HD 142666 are located at the lower left part of the box occupied by HAEBE stars close to the region occupied by Be stars. Thus their evolutionary status is unknown: are they PMS stars, or at a later stage of evolution? This conclusion applies also to the stars HD 35929, HD 132947, EW CMa, MCW 1264 and WRA 15-535. These stars are located outside the box occupied by HAEBE stars but their $\log \left[F_{60} / F_{25}\right]$ ratio is only an upper limit.

Besides, most of the above-mentioned stars show polarization at the level of about $1 \%$, which is in good agreement with the conclusions of Cote \& Waters (1987) and Waters \& Marlborough (1992). These authors have shown that an upper limit on the optical polarization of Be stars is about $1.5 \%$. In three cases the polarization is high $(4 \%$ $-5 \%$ for MWC 863, KK Oph and MWC 158). Note however that the polarimetric variability of these three stars is small, and most probably therefore a significant part of the observed polarization has an interstellar origin. For objects which are located well away from the positions of normal stars on the IRAS two-colour diagram the degree of polarization is statistically larger. The stars HD 37411, He 3-672, Hilt 641, HD 90578 and MWC 925 are located close to, or in the region of, planetary nebulae. We conclude that these objects also are probably not young.

On the other hand the stars HD 34282, V346 Ori and HD 141569 are located well away from the position of normal stars in the region of sources with evidence of matter outflows. Thus it is likely that they are very young. Note however that in our survey these 
Table 5. IRAS data for the programme stars. Colons denote that the $60 \mu \mathrm{m}$ flux is an upper limit

\begin{tabular}{|c|c|c|c|c|c|}
\hline Name & $\log \left[F_{25} / F_{12}\right]$ & $\log \left[F_{60} / F_{25}\right]$ & Name & $\log \left[F_{25} / F_{12}\right]$ & $\log \left[F_{60} / F_{25}\right]$ \\
\hline V1012 Ori & 0.69 & 0.17 & RY Lup & 0.29 & 0.29 \\
\hline HD 34282 & 0.37 & 0.80 & MWC 863 & 0.01 & -0.35 \\
\hline V346 Ori & 0.60 & 0.65 & KK Oph & -0.01 & -0.19 \\
\hline HD 35929 & -0.14 & $\leq-0.13:$ & HD 163296 & 0.06 & 0.13 \\
\hline RY Ori & 0.02 & $\leq 0.44:$ & $\mathrm{LkH} \alpha 112$ & & \\
\hline V586 Ori & -0.03 & $\leq 0.40$ & $\mathrm{LkH} \alpha 115$ & & \\
\hline HD 37357 & 0.14 & 0.02 & VV Ser & -0.09 & 0.23 \\
\hline HD 37411 & 0.47 & -0.14 & S CrA & 0.29 & 0.30 \\
\hline V599 Ori & 0.68 & 0.43 & V $1295 \mathrm{Aql}$ & -0.11 & -0.46 \\
\hline V350 Ori & 0.27 & -0.17 & HD 290770 & 0.14 & -0.02 \\
\hline MWC 120 & -0.07 & $\leq-0.26$ : & AS 117 & 0.37 & -0.14 \\
\hline MWC 137 & 0.37 & 1.01 & AS 119 & 0.04 & -0.64 \\
\hline VY Mon & 0.29 & 0.21 & MWC 158 & -0.05 & -0.67 \\
\hline MWC 148 & \multicolumn{2}{|c|}{ No IRAS data } & EW CMa & -0.17 & $\leq 0.12$ : \\
\hline GU CMa & 0.50 & $\leq 0.82$ & MWC 843 & 0.19 & 0.54 \\
\hline $\mathrm{LkH} \alpha 218$ & $\leq-0.49$ : & & AS 160 & -0.08 & -0.79 \\
\hline $\mathrm{Z} \mathrm{CMa}$ & 0.24 & 0.18 & 3 Pup & -0.24 & -0.76 \\
\hline NX Pup & 0.06 & 0.24 & Hilt 641 & 0.91 & $\leq 0.42:$ \\
\hline RCW 34 & 0.82 & 0.84 & Нe $3-140$ & -0.06 & -0.60 \\
\hline He $3-331$ & -0.04 & -0.62 & He $3-180$ & 0.27 & $\leq 1.93$ \\
\hline He $3-515$ & -0.14 & -0.11 & He 3-365 & 0.21 & 0.09 \\
\hline He $3-554$ & -0.12 & -0.68 & WRA $15-535$ & -0.48 & $\leq 0.39$ : \\
\hline Нe $3-597$ & 0.44 & 0.24 & HD 90578 & 0.65 & $\leq 0.20$ \\
\hline He 3-644 & -0.17 & -0.64 & HD 303004 & 1.00 & 0.56 \\
\hline Нe $3-672$ & 0.57 & -0.17 & WRA 15-689 & & \\
\hline Не $3-692$ & -0.02 & -0.26 & MCW 1264 & -0.24 & $\leq 0.37:$ \\
\hline Нe 3-741 & -0.01 & -0.19 & MWC 257 & -0.07 & 1.44 \\
\hline HD 132947 & -0.28 & $\leq 0.34:$ & MWC 272 & 0.19 & $\leq 1.24$ : \\
\hline HD 141569 & 0.53 & 0.47 & MWC 900 & -0.26 & -0.79 \\
\hline HD 142666 & 0.12 & -0.19 & MWC 925 & 0.81 & 0.21 \\
\hline
\end{tabular}

objects were observed close to maximum light and the polarization detected was small. The stars MWC 137, RCW 34 and V 599 Ori are also located in the same region but show large polarization. Note however that MWC 137 and RCW 34 are both surrounded by $\mathrm{H}$ II regions and were studied earlier as PNe or protoplanetary nebulae (Jouain et al. 1990; Zijlstra et al. 1990). The stars MWC 272 and MWC 257 are located directly in the region occupied by IR sources in star forming regions but their classification as PMS stars is questionable (see Sect. 3).

\subsection{Variability of polarization}

Here we discuss statistically the polarimetric variability for the various groups of objects. For this aim we select stars for which one or more pairs of repeated measurements (on a different time-scale) were obtained. To study the variability statistically we separated the stars into three groups: HAEBE stars, Be and B[e] stars, taking into account their IRAS data and previously published classification. The number of stars for which there is a suggestion of rapid ( $\lesssim 1$ hour) polarimetric variability is 30 .
Analysis of our data allows us to conclude that there is no significant difference in the proportion of stars showing rapid variability between the three classes above: such variability is present in about $40 \%$ of stars in each of the three classes. Thus, for approximately half of investigated HAEBE, Be and B[e] stars Thomson scattering can provide a detectable influence on the observed polarimetric variability.

We note that, in some HAEBE and $\mathrm{B}[\mathrm{e}]$ stars, rapid variations may also of course be produced by dust illumination effects: for example, rapid polarization variations might occur as a result of scattering by dust grains in the circumstellar shell of a star showing rapid photometric variability. However in this case one would usually expect corresponding changes in photometry, which is not the case for most of our programme stars. To really distinguish between mechanisms of rapid polarimetric variability one must carry out a detailed study of the variability of the wavelength dependence of polarization, and combine this with precise contemporaneous photometry.

Note however that in most investigated Be stars the amplitude of rapid variability is comparable with the value 
of polarization, whereas for at least half of the $\mathrm{B}[\mathrm{e}]$ and HAEBE stars detected the polarization is much larger than the amplitude of rapid variability. Therefore, a high level of polarization is due to scattering by dust grains in their CS shells or, in some cases, to a large interstellar component. Further, variability on a time-scale of days was detected in most of the investigated stars for which such an investigation could be carried out.

An analysis of our data, together with polarimetric data available in the literature, allows us to investigate the variability on longer time-scales ( 21 year) for RCW 34, MWC 272, Z CMa, V586 Ori, MWC 925 and He 3-365. Significant polarimetric variability was not present in only eight stars from our survey (MWC 120, MWC 137, Z CMa, RCW 34, He 3-140, HD 303004, WRA 15-689 and MWC 900). However polarimetric variability for two of these stars (Z CMa and MWC 137) has been claimed earlier in the literature and variability on a long time-scale does occur in RCW 34.

Thus polarimetric variability on a range of time-scales is present in most of the investigated stars, in all three classes. This is in good agreement with the results obtained by Menard \& Bastien (1992) for young TTS. They noted that "the vast majority (maybe as many as 100\%) of all TTS with enough measurements are polarimetric variables". However, the presence of any kind of variability cannot be a definite criterion for selecting objects for membership of the various groups mentioned above.

\subsection{Presence of intrinsic polarization}

As with Zickgraf \& Schulte-Ladbeck (1989), we suppose that an intrinsic component of polarization in an object is present if at least one of the following criteria holds:

(a) $p_{\text {obs }}(\lambda)$ is variable on different time-scales;

(b) $p_{\text {obs }}(\lambda)$ shows a wavelength dependence which is significantly different from the Serkowski et al. (1975) law for interstellar polarization;

(c) the PA varies significantly with $\lambda$.

The presence of polarimetric variability allows us to deduce the existence of an intrinsic component of polarization in most of our programme stars (except for stars with insufficient data, and objects for which polarization was not detected). Only five stars do not show any evidence of polarimetric variability on different timescales (MWC 120, He 3-140, HD 303004, WRA 15-689 and MWC 900).

\subsection{Circular polarization}

To date, circular polarization for HAEBE stars has been measured for very few objects (see Menard et al. 1988 for R Mon, Voshinnikov et al. (1988) for UX Ori, Hutchinson et al. (1994) for a group of 10 HAEBE stars). Menard et al.
(1988) have discussed their data for R Mon in terms of multiple scattering in an accretion disc and have pointed out that the data cannot be explained by a model with aligned grains. Voshinnikov et al. (1988) have detected circular polarization (at the $5 \sigma$ level) for the HAEBE star UX Ori - which shows Algol-like minima - of about $1 \%$ in one of the light curve minima. In the egress from the second minimum they also detected an increase in circular polarization at the level of $2-2.5 \sigma$. Between minima the circular polarization was close to zero. They have interpreted this in terms of a model with aligned dust grains. Later measurements of circular polarization for a group of HAEBE stars were carried out by Hutchinson et al. (1994). They detected circular polarization (at the $3 \sigma$ level) on a single occasion, in a single waveband, in AK Sco, HD 97048, MWC 863, MWC 272 and HD 144776. No circular polarization was detected at the $3 \sigma$ level for HAEBE stars V586 Sco, V380 Ori, V1295 Aql, BF Ori and UX Ori. Note however that they observed UX Ori only at maximum light.

In our survey circular polarization at the $3 \sigma$ level was detected on a single occasion in MWC 120, RCW 34, He 3554, HD 37411, twice in LkH $\alpha 115(4 \sigma)$ and twice in He 3$365(3 \sigma)$. Polarimetric observations with good accuracy of HD 163296 show circular polarization twice in the $V$ band $(3 \sigma)$, three times in the $R$ band (two to $3 \sigma$ and one to $8 \sigma$ ) and seven times in the $I$ band (six to $3 \sigma$ and one to $5 \sigma$ ). Note however that the detection of circular polarization for MWC 120, RCW 34, He 3-554, HD 37411 and He 3365 was not statistically significant. For HD 163296 the presence of circular polarization was more significant (see Fig. 25) but according to Beskrovnaya et al. (1998) the main mechanism of polarization observed in HD 163296 is scattering on azimuthal inhomogeneities forming in different CS regions of a gaseous envelope.

If we consider the scattering of non-polarized light, averaging over all orientations of a scattering particle leaves only linear polarization. To determine circular polarization one needs to have at least three physical vectors. In other words, the presence of circular polarization requires an asymmetry in the scattering particles' distribution (i.e. non-spherical shells) and aligned grains simultaneously, or the presence of at least two ensembles of oriented scattering particles with different orientation (see Dolginov et al. 1995 for details). For the case of transforming linear polarization $\left(p_{1}\right)$ to circular polarization $\left(p_{\mathrm{c}}\right)$, the latter is limited by the following equation:

$p_{\mathrm{c}} \leq p_{\mathrm{l}}^{2}$.

If an object shows linear polarization of about $1 \%$, the circular polarization can not be much more than $0.01 \%$. Thus, to definitely detect circular polarization (at least at a $3 \sigma$ level) which arises from the conversion of linear to circular polarization, the polarization accuracy has to be of the order of $0.003 \%$. From our data we conclude that the estimates of circular polarization obtained here are only 


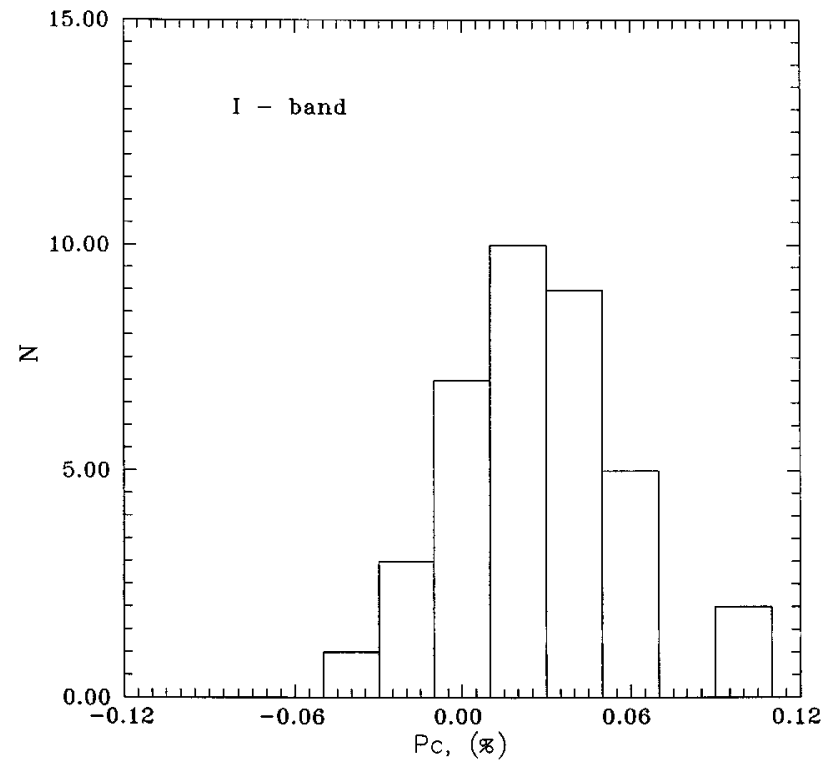

Fig. 25. Histogram of circular polarization distribution for HD 163296 in the $I_{\mathrm{c}}$ band

upper limits. This does not allow us to conclude whether multiple scattering, or scattering by aligned dust grains, may or may not play a rôle in the polarization of HAEBE stars and other stars from our programme. However most of the stars in our survey are bright objects, with linear polarization that is relatively low, whereas for faint stars with large linear polarization (MWC 137, V599 Ori, RY Ori, VY Mon, RCW 34 etc.) the observational errors were large. More precise observations of circular polarization using larger telescopes are highly desirable.

In addition, we note that circular polarization was detected at a statistically significant level for the TTS RY Lup, mainly at minimum light (in the $V$ band twice to $3 \sigma$, once to $4 \sigma$ and once to $7 \sigma$; in the $R_{\mathrm{c}}$ band twice to $3 \sigma$; in the $I_{\mathrm{c}}$ band once to $7 \sigma$ ). This fact is not surprising as, according to Bastien et al. (1988), circular polarization was detected in most investigated TTS and is attributed to multiple scattering in the CS envelopes. Note that Hutchinson et al. (1994) have claimed the nondetection of circular polarization in RY Lup in their study to a $5 \sigma$ (or even $3 \sigma$ ) level. However their polarimetric observations were carried out mainly through a clear filter but, as follows from our data (see for example HD 163296), circular polarization may have strong wavelength dependence. Note that, in the case of RY Lup $p_{\mathrm{c}}$ exceeds $p_{1}^{2}$. Thus, the circular polarization cannot be due to conversion from linear polarization and a likely mechanism for its origin is scattering by oriented dust grains in the circumstellar envelope.

\section{Relationship between the polarimetric and photometric data}

\subsection{Polarization and optical photometry}

Only four stars in our sample show a clear correlation between brightness and polarimetry. These are V350 Ori (probably a HAEBE star with Algol-like minima), VY Mon (a HAEBE star or FU Ori type star), the peculiar star WRA 15-535 and the TTS RY Lup; in these cases the polarization was larger when the star was faint. For other stars which show polarimetric variability these variations are not accompanied by changes in brightness. However if the dominant source of photometric variability in HAEBE stars with Algol-like minima is variable extinction (i.e. obscuration by proto-planetary clouds) then the requirement that such systems are only observed with their discs nearly edge-on for all such systems is highly unlikely. Note however that some objects were observed in our survey only at high (or low) levels of brightness. However we know that the photometric amplitudes for many of these stars are large (see Tables 1 and 2). Thus in order to draw any conclusions on the correlation between the photometry and polarization additional observations at a different light curve levels are needed.

\subsection{Polarization and near IR excesses}

As mentioned in Sect. 1 the correlation between polarization and IR excess was established for young TTS by Bastien $(1982,1985)$ and for HAEBE and TTS by Yudin (1988) in the sense that stars with a large IR excess also have large polarization. This fact suggests that the IR excess in young stars, as well as the polarization, are produced by the same agent. While it is now well established that dust grains are responsible for IR excess longward of the $L(3.5 \mu \mathrm{m})$ band, the observed polarization in young stars in many cases is also connected with scattering on the dust grains. Note that the data obtained here indicate that circular polarization was not statistically detected in most of the early type stars in our survey. Therefore we can refute the suggestion that multiple scattering, or scattering on aligned nonspherical dust grains, is a significant process in the CS shells of at least some of the investigated stars having large detected polarization. Furthermore, in most of the stars with large polarization, it is likely that the contribution of interstellar polarization is large.

To explain large values of polarization by the presence of dust envelopes in terms of single scattering we must suppose that the geometrical distribution of grains is not spherical, i.e. the dust is concentrated mostly in CS discs. But in this case large values of polarization will be observed only if the dust discs are have large inclination to the line of sight. While we cannot assert that all the observed stars have dust discs which are oriented edge-on 
there will be some stars with small polarization and large IR excesses but not vice versa. The latter case (large polarization and a small IR excess) may occur only if the contribution of interstellar polarization is large, or an additional mechanism of polarization is at work. For example, as was shown by Yudin (1988), classical Be stars do not fit the relation on the $\log p-(V-L)_{*}$ diagram obtained for young stars and in general they have small IR excess due to the dust (in the $L$ band) but show polarization at the $1 \%-2 \%$ level. This leads to the possibility of discriminating between stars that are undergoing PMS evolution and stars at later stages of evolution.

It is well known that the linear polarization of Be stars has been interpreted in terms of electron scattering by free CS electrons whose distribution is not spherically symmetrical (Waters \& Marlborough 1992). With such a model it has been shown that it is not easy to produce a degree of optical polarization above $\approx 1.5 \%$, which is indeed is about the maximum degree of polarization observed in Be stars (see McLean \& Brown 1978; Poeckert et al. 1979). Waters \& Marlborough (1992) have pointed out that, for a model in which the optical depth for electron scattering is large, multiple scattering will tend to reduce the degree of observed polarization. Note however that there is no evidence for circular polarization in most of the stars in our survey and that there is therefore no evidence for multiple scattering in their CS shells. Some Be stars in the relation derived by Yudin (1988) and which show polarization above the $1.5 \%$ limit probably have an additional component of polarization, such as scattering by grains, or interstellar polarization. In addition Waters \& Marlborough (1992) have claimed that the bulk of the linear polarization is produced in layers within 2 or 3 stellar radii,which can produce polarimetric variability on a time-scale of few minutes due to illumination effects. This conclusion is in good agreement with the results obtained here.

Combination of near IR continuum observations and optical linear and circular polarization may provide constrains on (i) the geometrical distribution of scatterers, (ii) selection of stars at different stages of evolution and (iii) the different mechanisms of polarization. This conclusion can be applied to HAEBE and Be stars, but we can not discriminate between HAEBE and $\mathrm{B}[\mathrm{e}]$ stars by using this method, as near IR excesses in both classes of stars are explained by the presence of hot dust. Moreover, according to Zickgraf \& Schulte-Ladbeck (1989) and Magalhaes (1992) in some cases dust is the main polarizing agent in $\mathrm{B}[\mathrm{e}]$ stars envelopes. Nevertheless, as was shown above, such a separation can be made by using the IRAS twocolour diagram.

To obtain additional information on the possible classification of programme stars, and to estimate roughly the inclination of the dust discs around them, we collect available data on the IR photometry in the $L$ band (see Tables 1 and 2). Note that Yudin (1988) has found a significant $(k=0.94)$ correlation between polarization in

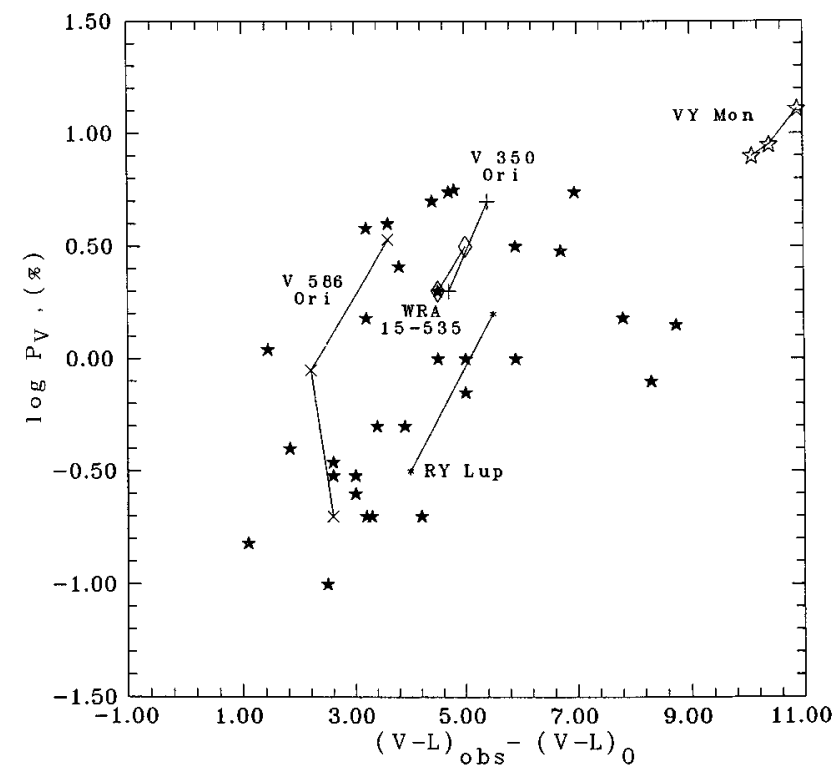

Fig. 26. Polarization-photometry diagram for the objects from our survey

the $R$ band and the optical-IR colour excess $(V-L)_{*}$, where $(V-L)_{*}=(V-L)_{\text {obs }}-(V-L)_{0}-\left(A_{V}-A_{L}\right)$; here $(V-L)_{0}$ is the normal colour index corresponding to the spectral class of the star, and $\left(A_{V}\right.$ and $\left.A_{L}\right)$ are the interstellar extinction in the respective photometric bands. It is easy to show that $(V-L)_{*}$ determines the relative contribution of the CS shell to the IR emission in comparison with the stellar photosphere in the given IR photometric band (Yudin 1988). In our study we have obtained numerous polarimetric data, mainly in the $V$ band. Since the values of polarization in the $V$ and $R$ bands are often strongly correlated (see for example Bastien (1985), and since our data also suggest a strong correlation between $p_{V}$ and $p_{R_{\mathrm{c}}}$, we can use the polarimetric data obtained in the $V$ band, which are more numerous. More significant is the fact that we have estimates of interstellar extinction for only 19 stars in our survey, whereas the IR data are available for 36 of the programme stars. Note however that a significant correlation of polarization with $(V-L)_{\text {obs }}-(V-L)_{0}$, or even with $(V-L)_{\text {obs }}$, was also established for young stars, but the correlation coefficient is smaller $(k \approx 0.7)$. Therefore we construct for the objects from our survey two diagrams, namely $\log p_{V}-(V-L)_{*}$ and log $p-\left\{(V-L)_{\text {obs }}-(V-L)_{0}\right\}$ (see Figs. 26, 27).

The following conclusions can be drawn from the analysis of the location of the programme stars on the above mentioned diagrams.

1. For most objects there is a tendency to show large polarization with large near IR excess.

2. Some stars, such as He 3-672, EW CMa, HD 37411, He 3-331, HD 37357, MWC 158, MWC 148, AS 160, $\mathrm{LkH} \alpha 218$, do not fall on the dependence obtained for young stars, and some of them are located in the field 


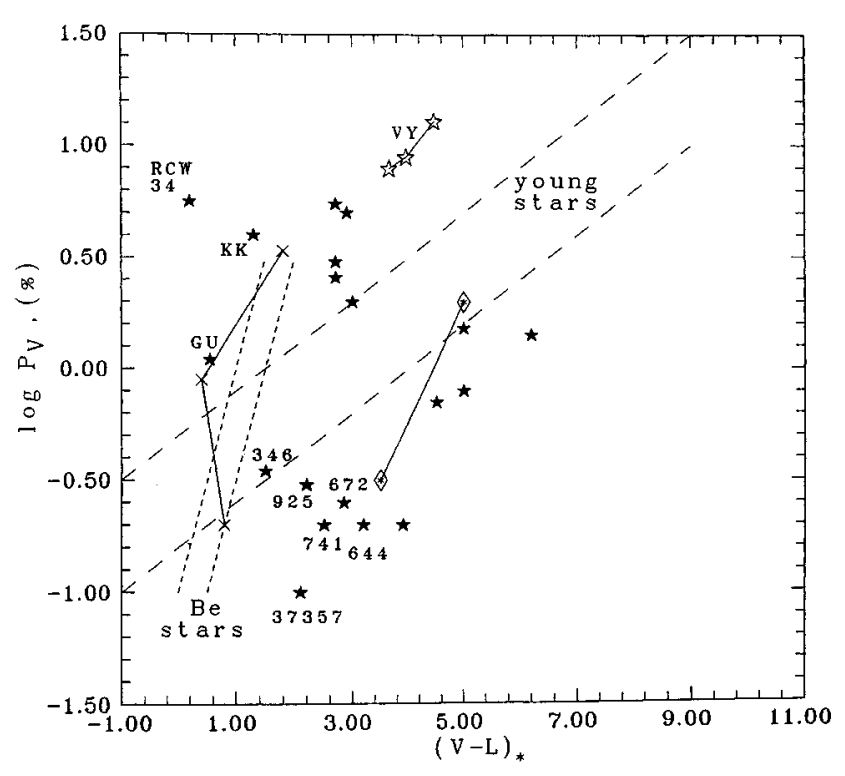

Fig. 27. Polarization-near IR excess diagram for the objects from our survey

occupied by Be stars. Thus it is likely that they are not young objects, or are at a stage of evolution close to the main sequence, in good agreement with our conclusion based on an analysis of the IRAS two-colour diagram (see above).

3. The stars V350 Ori, VV Ser, S CrA, V599 Ori, RY Ori, NX Pup, He 3-365, WRA 15-535 all fall approximately on the dependence derived for young stars.

4. The locus of VY Mon on the diagram due to the variability of polarization and visual flux takes place parallel to, but above, the dependence relation for young stars, suggesting the presence of a large component of interstellar polarization $(\approx 5 \%)$.

5. The locus of V350 Ori and WRA $15-535$ on the diagram takes place in the strip occupied by the majority of young stars.

6. The position of He 3-597, MWC 137, MWC 272, MWC 863, together with the presence of a large but nonvariable component of polarization, also suggests a significant component of interstellar polarization.

7. The position of RCW 34 is peculiar. This O-type star shows a small near IR excess, together with large polarization; however its far IR excess is large. Taking into account data obtained by Jain et al. (1995) we can deduce the presence of an interstellar component of polarization at the level of $\approx 3 \%$, but even in this case the intrinsic polarization would exceed $2.5 \%$.

As noted in Sect. 3, five stars in our sample which are binary (Z CMa, NX Pup, MWC 863, KK Oph and $\mathrm{S}$ CrA) show the PA of observed polarization close to the PA between the components. If the components in the above mentioned systems are physically connected and at least one of the components is surrounded by a CS disc then such a disc must be oriented in the direction be- tween the components. In all the cases mentioned above the observed polarization is parallel (or close) to the disc plane. However, it is well known that for optically thin discs the polarization has a direction perpendicular to the disc plane (see for example Whitney \& Hartmann 1992). According to Whitney \& Hartmann (1993) "for optically thick envelopes, with rotation or wind-driven holes the net system polarization is parallel to the disc plane since more singly scattered light escapes from the polar regions". We conclude that the observed behavior (at least for KK Oph and MWC 863) can be explained in terms of the model discussed by Whitney \& Hartmann (1993). On the other hand for the stars V350 Ori, He 3-672, HD 132947 and RY Lup the data on the $U-Q$ diagram are concentrated along a line that is also evidence of non-spherically symmetrical envelopes.

\section{Conclusions}

The polarimetric (linear and circular) observations of a large group (60 objects) of peculiar early-type stars (Herbig Ae/Be stars and candidate members, B [e] stars, extreme emission line objects etc.) have been presented. For 40 out of the 60 objects polarization was measured for the first time. However it would be most interesting to obtain further polarimetric data for the stars in this programme at different photometric levels.

Our main conclusions may be summarized as follows:

1. No polarization was detected, at the $95 \%$ confidence level, for 5 out of the 60 stars (V1012 Ori, HD 34282 , HD 35929, HD 37357, and HD 290770). For V346 Ori, RY Ori, V599 Ori, LkH $\alpha 112$ and AS 117, polarization at the $95 \%$ confidence level was detected on a single occasion.

2. There was no evidence for polarimetric variability in only 8 stars in our survey (MWC 120, MWC 137, Z CMa, RCW 34, He 3-140, HD 303004, WRA 15-689 and MWC 900) but polarimetric variability for two of these stars (Z CMa and MWC 137) has been claimed in the literature; variability on a long time-scale (years) may take place in RCW 34.

3. Analysis of the data allows us to conclude that there is no significant difference in the proportion of stars showing rapid (on a time-scale of minutes) variability between the three classes of stars (HAEBE, Be and $\mathrm{B}[\mathrm{e}])$. Such variability is present in about $40 \%$ of stars in the each class for which rapid variability has been studied. However, in most of the Be stars investigated the amplitude of rapid variability is comparable with the value of polarization, whereas for at least half of the $\mathrm{B}[\mathrm{e}]$ and HAEBE stars the detected polarization is much larger than the amplitude of rapid variability.

4. Variability on a time-scale of days was detected in most of the programme stars for which such an investigation was carried out. Probably the vast majority (maybe as 
many as $100 \%$ ) of all HAEBE stars, as well as Be stars with enough measurements, are polarimetric variables.

5. Our data, together with the polarimetric data in the literature, enables us to deduce variability on a long time-scale (year or longer) for RCW 34, MWC 272, Z CMa, V586 Ori, MWC 925 and He 3-365.

6. Consequently variability on different time-scales is present in most of the investigated stars, in all three classes, and cannot be used as a defining criterion for the classifying stars in the various groups.

7. We deduce the presence of an intrinsic component of polarization in most of the investigated stars (except for small number of the stars with insufficient data, and stars for which polarization was not detected). Only five stars do not show evidence of polarimetric variability on different time-scales (MWC 120, He 3140, HD 303004, WRA 15-689 and MWC 900) but for most of them the wavelength dependence of polarization is unlike that of interstellar polarization.

8. In our survey circular polarization at $\geq 3 \sigma$ level was detected in MWC 120, RCW 34, He 3-554, HD 37411 , $\mathrm{LkH} \alpha 115$ and He 3-365. Polarimetric observations with a good accuracy of HD 163296 showed the presence of circular polarization. However we detected no significant circular polarization in MWC 120, RCW 34, He 3-554, HD 37411 and He 3-365.

9. From the above we conclude that circular polarization has not been detected (within the errors) in most of the HAEBE stars and candidate members in our survey. This does not allow us to conclude whether or not multiple scattering or scattering by aligned dust grains play a rôle in the polarization of HAEBE stars and other stars in our programme. In addition we note that circular polarization was also detected at a statistically significant level for the TTS RY Lup, mainly at minimum light. This is in good agreement with the results of Bastien et al. (1988), that circular polarization was detected in most of their investigated TTS; it is attributed to multiple scattering in its CS shells.

10. Only four stars in our survey show a clear correlation between photometric state and polarimetry. These are V350 Ori (probably a HAEBE star with Algol-like minima), VY Mon (a HAEBE star or FU Ori type star), the peculiar star WRA 15-535 and the TTS RY Lup; for these objects the polarization was larger when the star was faint. For other stars which showed polarimetric variability the variations are not accompanied by photometric changes.

11. For most of the programme stars there is a tendency to show large polarization with large near-IR excess. Analysis of a star's position on the log $p_{V}-(V-L)_{*}$ diagram allows us to roughly select stars according to their evolutionary status. Conclusions on the evolutionary status of programme stars were also made from an analysis of the IRAS two-colour diagram. The intersection of the sets of objects identified from the analy- sis of the above two diagrams leads us to conclude that the stars EW CMa, HD 36929, He 3-331, He 3-554, He 3-692, AS 160, MWC 158, MWC 900, LkH $\alpha$ 218, 3 Pup and MCW 1264 are probably not young, and that the stars HD 37357, V586 Ori, He 3-515, HD 142666, MWC 863 probably lie close to the main sequence.

Acknowledgements. We thank the allocation committee of the SAAO for generous allocations of observing time, and the United Kingdom Particle Physics and Astronomy Research Council for the provision of travel funds. We also thank Dr. D. Buckley (SAAO) and the staff SAAO Sutherland for their support during the observations, and the referee (Dr P. Bastien) for his helpful comments. The data were reduced on the Keele University STARLINK node. We also acknowledge the use of the SIMBAD database. RVYu was supported in part by ESO grant A-07-022, INTAS grant 93-2478 and RFFR 96-02-19179.

\section{References}

Allen D.A., 1973, MNRAS 161, 145

Allen D.A., Swings J.P., 1976, A\&A 47, 293

Baier G, Keller E., Mundt R., Weigelt G., 1985, A\&A 153, 278

Balona L.A., 1995, MNRAS 277, 1547

Barbier R., Swings J.P., 1982, Be stars. Symp. IAU 98, Jaschek M., Groth M.G. (eds.). Dordrecht. D. Reidel Publ. Company, p. 103

Bastien P., 1982, A\&AS 48, 153; 48, 513

Bastien P., 1985, ApJS 59, 277

Bastien P., Menard F, 1988, ApJ 326, 334

Bastien P., Robert C., Nadeau R., 1989, ApJ 339, 1089

Berdyugin A.V., Berdyugina S.V., Grinin V.P., Minikulov N.H., 1990, Sov. Astron 34, 408

Berdyugin A.V., Grinin V.P., Minikulov N.H., 1992, Bull. Crimean Astrophys. Obs. 86, 65

Berdyugin A.V., et al., 1993, Bull. Crimean Astrophys. Obs. 87,98

Bergner Yu.K., Miroshnichenko A.S., Yudin R.V., et al., 1987, SvA Lett. 13, 84

Bergner Yu.K., Miroshnichenko A.S., Yudin R.V., et al., 1993, Peremennye zvezdy 23, 163

Bergner Yu.K., Miroshnichenko A.S., Yudin R.V., et al., 1995, A\&AS 112, 221

Berrilli F., Ceccarelli C., Liseau R., et al., 1989, MNRAS 237, 1

Berrilli F., Corciulo G., Ingrosso G., et al., 1992, ApJ 398, 254

Beskrovnaya N.G., Pogodin M.A., Najdenov I.D., et al., 1995, A\&A 298, 585

Beskrovnaya N.G., Pogodin M.A., Yudin R.V., et al., 1998, A\&AS 127, 243

Bjorkman K.S., Schulte-Ladbeck R.E., 1994, in: "The nature and evolutionary status of Herbig Ae/Be stars", The P.S., Perez M.R., van den Heuvel E.P.J. (eds.), ASP Conf. Ser. 62, p. 74

Blondel P.F.C., Tjin A Djie H.R.E., 1994, in: "The nature and evolutionary status of Herbig Ae/Be stars", The P.S., Perez M.R., van den Heuvel E.P.J. (eds.), ASP Conf. Ser. 62, p. 211 
Bogaert E., Waelkens C., 1990, in: "The infrared spectral region of stars", Jaschek C. and Andrillat Y. (eds.). Cambridge Univ. Press, p. 345

Breger M., 1976, ApJ 204, 789

Campbell A., Person S.E., Mathhews K., 1989, AJ 98, 643

Catala C., 1994, in: "The nature and evolutionary status of Herbig Ae/Be stars", The P.S., Perez M.R., van den Heuvel E.P.J. (eds.), ASP Conf. Ser. 62, p. 91

Chelli A., Cruz-Gonzalez I., Reipurth B., et al., 1985, A\&A 153,278

Clarke D., Schwartz H.E., Stewart B.G., 1985, A\&A 145, 232

Clarke D., Stewart B.G., 1986, Vistas Astron. 29, 27

Cote J., Waters L.B.F.M., 1987, A\&A 176, 93

Covino E., Terranegra L., Vittone A.A., Russo G., 1984, AJ 89, 1868

Coyne G.V., Gehrels T., Serkowski K., 1974, AJ 79, 581

Cropper M., 1985, MNRAS 212, 709

Dachs J., 1982, Be stars. Symp. IAU 98, Jaschek M., Groth M.G. (eds.). Dordrecht, D. Reidel Publ. Company, p. 19

Dolginov A.Z., Gnedin Yu.N., Silant'ev N.A., 1995, Propagation and polarization of radiation in Cosmic Media. Gordon and Breach Publishers

Drilling G.S., 1991, ApJS 76, 1033

Drissen L., Bastien P., St.-Louis N., 1989, AJ 97, 814

Dzhakusheva K.G., Kuratov K.S., Mukanov D.B., et al., 1988, SvA Lett. 14, 317

Eisloffel J., Ray T.P., 1994, in: "The nature and evolutionary status of Herbig Ae/Be stars", The P.S., Perez M.R., van den Heuvel E.P.J. (eds.), ASP Conf. Ser. 62, p. 384

Finkenzeller U., Mundt R., 1984, A\&AS 55, 109

Gahm G.F., Ahlin P., Lindroos K.P., 1983, A\&AS 51, 143

Garrison M.J., Anderson C.M., 1978, ApJ 221, 601

Gledhill T.M., Scarrott S.M., 1989, MNRAS 236, 139

Gnedin Yu.N., Kiselev N.N., Pogodin M.A., et al., 1992, SvA Lett. 18, 182

Grady C.A., Perez M.R., The P.S., 1994, in: "The nature and evolutionary status of Herbig Ae/Be stars", The P.S., Perez M.R., van den Heuvel E.P.J. (eds.), ASP Conf. Ser. 62, p. 409

Graham J.A., 1992, PASP 104, 479

Grankin K.N., 1995, PhD thesis, Pulkovo Observatory

Grinin V.P., Kiselev N.N., Minikulov N.H., Chernova G.P., 1988, SvA Lett. 14, 219

Grinin V.P., 1992, Astron. Astroph. Transact. 3, 17

Grinin V.P., 1994, in: "The nature and evolutionary status of Herbig Ae/Be stars", The P.S., Perez M.R., van den Heuvel E.P.J. (eds.), ASP Conf. Ser. 62, p. 63

Hall J.S., 1958, Publ. US Naval Obs. 17, pt. VI

Harris S., Clegy P., Hugnes J., 1988, MNRAS 235, 441

Heckert P.A., Zeilik M., 1981, AJ 86, 1076

Henning Th., Launhardt R., Steinacker J., Thamm E., 1994, A\&A 291, 546

Herbig G., 1960, ApJS 4, 337

Herbig G., 1994, in: "The nature and evolutionary status of Herbig Ae/Be stars", The P.S., Perez M.R., van den Heuvel E.P.J., ASP Conf. Ser. 62, p. 3

Herzog A.D., Gehrz R.D., Hachwell J.A., 1980, ApJ 236, 189

Hillenbradt L.A., Strom S.E., Vrba F.J., Keene J., 1992, ApJ 397, 613

Hiltner W.A., 1956, ApJS 2, 389

Hsu J.C., Breger M., 1982, ApJ 262, 732
Hutchinson M.G., Albinson J.S., Barrett P., et al., 1994, A\&A 285, 883 (HAB94)

Jensen E.L.N., Mathieu R.D., Fuller G.A., 1996, ApJ 458, 312

Jian S.K., Bhatt H.C., Sagar R., 1990, A\&AS 83, 237

Jian S.K., Bhatt H.C., 1995, A\&AS 111, 399 (JB95)

Jourain De Muizon M., Cox P., Lequeux J., 1990, A\&AS 83, 337

Kaufl H.U., Renzini A., Stanghellini L., 1993, ApJ 410, 251

Kilkenny D., Whittet D.C.B., Davies J.K., et al., 1985, SAAO Circ. 9, 55

Kholopov P.N., 1985, General Catalogue of Variable Stars. The fourth edition, Moskow, Nauka

Kolotilov E.A., 1991, Sov. Astron. Lett. 17, 144

Leinert C., 1986, A\&A 155, L6

Lopes D.F., Neto A.D., de freitas Pacheco J.A., 1992, A\&A 261,482

Magalhaes A.M., 1992, ApJ 398, 286

Manfroid J., Sterken C., Bruch A., et al., 1991, ESO Scientific Report, No. 8

Manfroid J., Sterken C., Cunow B., et al., 1994, ESO Scientific Report, No. 14

Mannings V., 1994, MNRAS 271, 587

Martin P.G., Adamson A.J., Whittet D.C.B., et al., 1992, ApJ 392, 691

Martin E.L., 1994, in: "The nature and evolutionary status of Herbig Ae/Be stars", The P.S., Perez M.R., van den Heuvel E.P.J. (eds.), ASP Conf. Ser. 62, p. 315

McGregor P.J., Hyland A.R., Hillier D.J., 1988, ApJ 324, 1071

McGregor P.J., Harrison T.E., Hough J.H., Bailey J.A., 1994, MNRAS 267, 755

McLean I.S., Brown J.C., 1978, A\&A 69, 291

Menard F., Bastien P., Robert C., 1988, ApJ 335, 290

Menard F., Bastien P., 1992, AJ 103, 564

Miroshnichenko A.S., Yudin R.V., Yakubov S.D., 1992, Astron. Astrophys. Trans. 1, 211

Miroshnichenko A.S., Yudin R.V., 1993, Proc. VI International Workshop of OAC. "Stellar Jets and Bipolar Outflows", 1991, Errico L. and Vittone A.A. (eds), p. 173

Miroshnichenko A.S., Yudin R.V., Shejkina T.A., Turdaliev B., 1993, IBVS No. 3937

Miroshnichenko A.S., Bergner Yu.K., Kuratov K.S., 1997, Astron. Lett. 23, 97

Mitskevich A.S., 1995, A\&A 298, 231

Molster F.J., The P.S., 1994, in: "The nature and evolutionary status of Herbig Ae/Be stars", The P.S., Perez M.R., van den Heuvel E.P.J. (eds.), ASP Conf. Ser. 62, p. 199

Naghizadeh-Khouei J., Clarke D., 1993, A\&A 274, 968

Odenwald S.F., 1989, AJ 97, 801

Oudmaijer R.D., van der Veen W.E.C.J., Waters L.B.F.M., et al., 1992, A\&AS 96, 625

Palla F., Stahler S.W., 1990, ApJ 360, L47

Pavlova L.A., Rspaev F.K., 1985, Astrophys. 23, 521

Petrova N.N., Shevchenko V.S., 1987, SvA Lett. 13, 289

Poeckert R., Bastien P., Landstreet J.D., 1979, AJ 84, 812

Pogodin M.A., 1997, A\&A 317, 185

Reipurth B., Zinnecker H., 1993, A\&A 278, 81

Scholler M., Brandner W., Lehmann T., Weigelt G., Zinnecker H., 1996, A\&A 315, 445

Serkowski K., 1958, Acta. Astron. 8, 135

Serkowski K., 1962, Advances in Astronomy and Astrophysics 1, Z. Kopal (ed.). Academic Press, New York, London 
Serkowski K., Gehrels T., Wisniewski W., 1969, AJ 74, 85

Serkowski K., Mathewson D.S., Ford V.L., 1975, ApJ 196, 261

Serkowski K., 1970, ApJ 160, 1083

Shevchenko V.S., 1989, Herbig Ae/Be stars, Fan, Tashkent

Shevchenko V.S., Grankin K.N., Ibragimov M.A., et al., 1994, in: "The nature and evolutionary status of Herbig Ae/Be stars", The P.S., Perez M.R., van den Heuvel E.P.J. (eds.), ASP Conf. Ser. 62, p. 57

Shore S.N., Brown D.N., Bopp B.W., et al., 1990, ApJS 73, 461

Scarrott S.M., Draper P.W., Warren-Smith R.F., 1989, MNRAS 237, 621

Skinner S.L., Brown A., Stewart R.T., 1993, ApJS 87, 217

Sterken C., Manfroid J., Anton K., et al., 1993, ESO Scientific Report, No. 12

Sterken C., Manfroid J., Beele D., et al., 1995, ESO Scientific Report, No. 16

Strom K.M., Newton G., Strom S.E., et al., 1989, ApJS 71, 183

Tamura M., Sato S., 1989, AJ 98, 1368

Tessier E., Bouvier J., Beuzit J.-L., Brandner W., 1994, ESO Messenger 78, 35

Teodorani M., Errico L., Vittone A.A., et al., 1998, Sov. Astron. (submitted)

The P.S., Perez M.R., van den Heuvel, 1994, "The nature and evolutionary status of Herbig Ae/Be stars", ASP Conf. Ser. 62

The P.S., de Winter D., Perez M.R., 1994, A\&A 104, 315

van der Veen W.E.C.J., Habing H.J., Geballe T.R., 1989, A\&A 226, 108

van der Veen W.E.C.J., Waters L.B.F.M., Trams H.E., Matthews A.A., 1994, A\&A 285, 551

Voshchinnikov N.V., Marchenko P.E., 1982, Sov. Astron. 26, 1115
Voschinnikov N.V., Grinin V.P., Kiselev N.N., Minikulov N.H., 1988, Astrophys. 28, 182

Voschinnikov N.V., Grinin V.P., 1991, Astrophys. 34, 84

Vrba F.J., 1975, ApJ 195, 101

Vrba F.J., Schmidt G.D., Hintzen P.M., 1979, ApJ 227, 185

Vrba F.J., Strom S.E., Strom K.M., 1987, AJ 96, 680

Waelkens C., Boraert E., Waters L.B.F.M., 1994, in: "The nature and evolutionary status of Herbig Ae/Be stars", The P.S., Perez M.R., van den Heuvel E.P.J. (eds.), ASP Conf. Ser. 62 , p. 405

Waters L.B.F.M., Cote J., Lamers H.J.G.L.M., 1987, A\&A 185, 206

Waters L.B.F.M., Malborough J.M., 1992, A\&A 256, 195

Weintraub D.A., 1992, ApJS 74, 575

Whittet D.C.B., Martin P.G., Hough J.H., et al., 1992, ApJ 386,562

Whitney B.A., Hartmann L., 1992, ApJ 395, 529

Whitney B.A., Hartmann L., 1993, ApJ 402, 605

Whitney B.A., Clayton G.C., Schulte-Ladbeck R.E., et al., 1993, ApJ 417, 687

Yudin R.V., 1988, Sov. Astron. 32, 652

Yudin R.V., 1990, in: "The infrared spectral region of stars", Jaschek C. and Andrillat Y. (eds.). Cambridge Univ. Press, p. 400

Yudin R.V., 1992, PhD Thesis, Pulkovo Observatory

Yudin R.V., 1994, in: "The nature and evolutionary status of Herbig Ae/Be stars", The P.S., Perez M.R., van den Heuvel E.P.J. (eds.), ASP Conf. Ser. 62, p. 82

Yudin R.V., 1995, in: IAU Colloquium No. 151 "Flares and Flashes", 1994, Greiner J., Duerbeck H.W., Gershberg R.E. (eds.), Lect. Notes Phys. 454, p. 227

Yudin R.V., 1996, A\&A 312, 234

Zickgraf F.-J., Schulte-Ladbeck R.E., 1989, A\&A 214, 274

Zijlstra A., Pottasch S., Bignell C., 1990, A\&AS 82, 273 